ESAIM: M2AN

Vol. 40, No 5, 2006, pp. 897-921

DOI: $10.1051 / \mathrm{m} 2 \mathrm{an}: 2006038$
ESAIM: Mathematical Modelling and Numerical Analysis

www.edpsciences.org/m2an

\title{
SPECTRAL ELEMENT DISCRETIZATION OF THE VORTICITY, VELOCITY AND PRESSURE FORMULATION OF THE STOKES PROBLEM
}

\author{
Karima Amoura ${ }^{1}$, Christine Bernardi ${ }^{2}$ And Nejmeddine Chorfi ${ }^{3}$
}

\begin{abstract}
We consider the Stokes problem provided with non standard boundary conditions which involve the normal component of the velocity and the tangential components of the vorticity. We write a variational formulation of this problem with three independent unknowns: the vorticity, the velocity and the pressure. Next we propose a discretization by spectral element methods which relies on this formulation. A detailed numerical analysis leads to optimal error estimates for the three unknowns and numerical experiments confirm the interest of the discretization.
\end{abstract}

Mathematics Subject Classification. 35Q30, 65N35.

Received: January 1st, 2006. Revised: June 13, 2006.

\section{INTRODUCTION}

We are interested in the spectral element discretization of the Stokes problem in a two- or three-dimensional bounded domain, when provided with boundary conditions on the normal component of the velocity and the vorticity in dimension 2 , on the normal component of the velocity and the tangential components of the vorticity in dimension 3. This type of boundary conditions occurs in a large number of flows, for instance for a fluid on both sides of a membrane, for water in a crack inside a rigid porous medium or when several approaches of turbulence are coupled. The formulation that we consider, first proposed in [13] and [18] (see also [1] and [14]) involves three unknowns, the vorticity, the velocity and the pressure. Even if the number of unknowns makes its discretization expensive, it seems to be the best adapted formulation for handling this type of boundary conditions. The first analysis of the corresponding variational problem is performed in [13] and [18] in the twodimensional case. We refer to [4], Section 2, for the extension to three-dimensional simply-connected domains and to [7], Section 2.5, for the treatment of multiply-connected domains. We only recall the main results proved in these works, in view of their discrete analogues.

The numerical analysis of discretizations of the Stokes problem relying on this formulation has first been performed for finite element methods, see [18] and the references therein. It has recently been extended to the case of spectral methods in [4], where the spaces of polynomials are the spectral analogues of the finite element

\footnotetext{
Keywords and phrases. Stokes problem; vorticity, velocity and pressure formulation; spectral element methods.

1 Université Badji-Mokhtar, Faculté des Sciences, Département de Mathématiques, B.P. 12, 23000 Annaba, Algeria. amouradz@yahoo.fr

${ }^{2}$ Laboratoire Jacques-Louis Lions, C.N.R.S. et Université Pierre et Marie Curie, B.C. 187, 4 place Jussieu, 75252 Paris Cedex 05, France. bernardi@ann.jussieu.fr

3 Département de Mathématiques, Faculté des Sciences de Tunis, Campus Universitaire, 1060 Tunis, Tunisia.

nejmeddine.chorfi@fst.rnu.tn
}

(c) EDP Sciences, SMAI 2007 
spaces introduced in [16]. We propose a discretization of this problem that relies on the spectral element method. We consider a partition of the domain into rectangles in dimension 2 or rectangular parallelepipeds in dimension 3 which is conforming and without overlap. The discrete spaces are constructed from tensorized spaces of polynomials of the same high degree on each subdomain as in [4], and some matching conditions are enforced on the interfaces between subdomains, in order to work with a conforming discretization. The discrete problem is then obtained by the Galerkin method with numerical integration.

We perform the numerical analysis of this discretization. This study combines the arguments introduced in [4] with some standard or less standard ideas of the spectral element method. We thus prove optimal error estimates for the three unknowns. It can be noted that this is a special property of the formulation that we use, since the approximation of the pressure for other formulations of the Stokes problem is most often non optimal (see [5], Sects. 24-26). We present some numerical experiments which confirm the optimality of the discretization.

An outline of the paper is as follows:

- In Section 2, we write the variational formulation of the problem in the case of homogeneous boundary conditions.

- Section 3 is devoted to the description of the spectral element discrete problem. We also prove its well-posedness.

- Optimal error estimates are derived in Section 4.

- In Section 5, we present some numerical experiments which turn out to be in good agreement with the analysis.

\section{The VELOCITY, VORTICITY AND PRESSURE FORMULATION}

Let $\Omega$ be a bounded connected domain in $\mathbb{R}^{d}, d=2$ or 3 , with a Lipschitz-continuous boundary $\partial \Omega$. The generic point in $\Omega$ is denoted by $\boldsymbol{x}=(x, y)$ or $\boldsymbol{x}=(x, y, z)$ according to the dimension $d$. We introduce the unit outward normal vector $\boldsymbol{n}$ to $\Omega$ on $\partial \Omega$ and we consider the Stokes problem

$$
\begin{cases}-\nu \Delta \boldsymbol{u}+\operatorname{grad} p=\boldsymbol{f} & \text { in } \Omega, \\ \operatorname{div} \boldsymbol{u}=0 & \text { in } \Omega, \\ \boldsymbol{u} \cdot \boldsymbol{n}=0 & \text { on } \partial \Omega, \\ \gamma_{t}(\operatorname{curl} \boldsymbol{u})=\mathbf{0} & \text { on } \partial \Omega .\end{cases}
$$

To make precise the sense of the operator $\gamma_{t}$, we recall that

- in dimension $d=2$, for any vector field $\boldsymbol{v}$ with components $v_{x}$ and $v_{y}, \operatorname{curl} \boldsymbol{v}$ stands for the scalar function $\partial_{x} v_{y}-\partial_{y} v_{x}$, so that the operator $\gamma_{t}$ is the trace operator on $\partial \Omega$;

- in dimension $d=3$, for any vector field $\boldsymbol{v}$ with components $v_{x}, v_{y}$ and $v_{z}, \operatorname{curl} \boldsymbol{v}$ stands for the vector field with components $\partial_{y} v_{z}-\partial_{z} v_{y}, \partial_{z} v_{x}-\partial_{x} v_{z}$ and $\partial_{x} v_{y}-\partial_{y} v_{x}$, and the operator $\gamma_{t}$ is the tangential trace operator on $\partial \Omega$, defined by: $\gamma_{t}(\boldsymbol{w})=\boldsymbol{w} \times \boldsymbol{n}$.

Of course, the operator $\gamma_{t}$ is only defined on smooth enough functions as will be made precise later on.

In system (2.1), the unknowns are the velocity $\boldsymbol{u}$ and the pressure $p$, while the data $\boldsymbol{f}$ represent a density of body forces. The viscosity $\nu$ is a positive constant. To go further, we introduce the vorticity $\boldsymbol{\omega}=\operatorname{curl} \boldsymbol{u}$ and observe that system (2.1) is fully equivalent to

$$
\begin{cases}\nu \operatorname{curl} \boldsymbol{\omega}+\operatorname{grad} p=\boldsymbol{f} & \text { in } \Omega, \\ \operatorname{div} \boldsymbol{u}=0 & \text { in } \Omega, \\ \boldsymbol{\omega}=\operatorname{curl} \boldsymbol{u} & \text { in } \Omega, \\ \boldsymbol{u} \cdot \boldsymbol{n}=0 & \text { on } \partial \Omega, \\ \gamma_{t}(\boldsymbol{\omega})=\mathbf{0} & \text { on } \partial \Omega .\end{cases}
$$


Note that the operator curl in the first line of this system coincides with the previous one in dimension $d=3$ while, in dimension $d=2$, it is applied to scalar functions $\varphi: \operatorname{curl} \varphi$ here denotes the vector field with components $\partial_{y} \varphi$ and $-\partial_{x} \varphi$.

However, as noted in [7], Section 2.2, the boundary conditions both in problems (2.1) and (2.2) are not sufficient to enforce the uniqueness of the solution in the case of a multiply-connected domain $\Omega$. Indeed, if $\left(\boldsymbol{\omega}_{1}, \boldsymbol{u}_{1}, p_{1}\right)$ and $\left(\boldsymbol{\omega}_{2}, \boldsymbol{u}_{2}, p_{2}\right)$ are two solutions of system (2.2), it can be checked that $\boldsymbol{\omega}_{1}-\boldsymbol{\omega}_{2}$ is zero. But the function $\boldsymbol{u}=\boldsymbol{u}_{1}-\boldsymbol{u}_{2}$ only satisfies

$$
\operatorname{curl} \boldsymbol{u}=\mathbf{0} \quad \text { and } \quad \operatorname{div} \boldsymbol{u}=0 \quad \text { in } \Omega, \quad \boldsymbol{u} \cdot \boldsymbol{n}=0 \quad \text { on } \partial \Omega .
$$

Examples of non-zero functions satisfying these three conditions are given explicitly in [2], Proposition 3.14. To make precise the further conditions that are needed for this uniqueness, we introduce some notation which are the same as in [2], Section 3.

Notation 2.1. Let $\Sigma_{j}, 1 \leq j \leq J$, be connected open curves or surfaces, called "cuts", such that

(i) each $\Sigma_{j}$ is an open part of a smooth manifold with dimension $d-1$;

(ii) each $\Sigma_{j}, 1 \leq j \leq J$, is contained in $\Omega$ and $\partial \Sigma_{j}$ is contained in $\partial \Omega$;

(iii) the intersection of $\Sigma_{j}$ and $\Sigma_{j^{\prime}}, 1 \leq j<j^{\prime} \leq J$, is empty;

(iv) the open set $\Omega^{\circ}=\Omega \backslash \cup_{j=1}^{J} \Sigma_{j}$ is simply-connected.

The existence of such $\Sigma_{j}$ is clear. We make the further assumption that the domain $\Omega^{\circ}$ is pseudo-Lipschitz, in the sense that, for each point $\boldsymbol{x}$ of $\partial \Omega^{\circ}$, the intersection of $\Omega^{\circ}$ with a smooth neighbourhood of $\boldsymbol{x}$ has one or two connected components and each of them has a Lipschitz-continuous boundary (we refer to [2], Sect. 3.a, for a more precise definition). Then, the further conditions read

$$
\langle\boldsymbol{u} \cdot \boldsymbol{n}, 1\rangle_{\Sigma_{j}}=0, \quad 1 \leq j \leq J
$$

where $\langle\cdot, \cdot\rangle_{\Sigma_{j}}$ stands for the duality pairing between $H^{-\frac{1}{2}}\left(\Sigma_{j}\right)$ and $H^{\frac{1}{2}}\left(\Sigma_{j}\right)$.

We introduce the domain $H(\operatorname{div}, \Omega)$ of the divergence operator, namely

$$
H(\operatorname{div}, \Omega)=\left\{\boldsymbol{v} \in L^{2}(\Omega)^{d} ; \operatorname{div} \boldsymbol{v} \in L^{2}(\Omega)\right\} .
$$

Since the normal trace operator: $\boldsymbol{v} \mapsto \boldsymbol{v} \cdot \boldsymbol{n}$ can be defined from $H(\operatorname{div}, \Omega)$ into $H^{-\frac{1}{2}}(\partial \Omega)$, see [15], Chapter I, Theorem 2.5, we also consider its kernel

$$
H_{0}(\operatorname{div}, \Omega)=\{\boldsymbol{v} \in H(\operatorname{div}, \Omega) ; \boldsymbol{v} \cdot \boldsymbol{n}=0 \text { on } \partial \Omega\} .
$$

Similarly, we introduce the domain of the curl operator

$$
H(\operatorname{curl}, \Omega)=\left\{\boldsymbol{\vartheta} \in L^{2}(\Omega)^{\frac{d(d-1)}{2}} ; \operatorname{curl} \boldsymbol{\vartheta} \in L^{2}(\Omega)^{d}\right\}
$$

The operator $\gamma_{t}$ is also defined on $H(\mathbf{c u r l}, \Omega)$ with values in $H^{\frac{1}{2}}(\partial \Omega)$ in dimension $d=2$ or in $H^{-\frac{1}{2}}(\partial \Omega)^{3}$ in dimension $d=3$, see [15], Chapter I, Theorem 2.11. So, we define the kernel

$$
H_{0}(\operatorname{curl}, \Omega)=\left\{\boldsymbol{\vartheta} \in H(\operatorname{curl}, \Omega) ; \gamma_{t}(\boldsymbol{\vartheta})=\mathbf{0} \text { on } \partial \Omega\right\} .
$$

It must be noted that the spaces $H(\operatorname{curl}, \Omega)$ and $H_{0}(\mathbf{c u r l}, \Omega)$ coincide with the spaces $H^{1}(\Omega)$ and $H_{0}^{1}(\Omega)$ in dimension $d=2$, but this is no longer true in dimension $d=3$. Finally, let $L_{0}^{2}(\Omega)$ stand for the space of functions in $L^{2}(\Omega)$ with a null integral on $\Omega$. 
In view of conditions (2.3) and according to [7], Section 2.5, we introduce the space

$$
\mathbb{D}(\Omega)=\left\{\boldsymbol{v} \in H_{0}(\operatorname{div}, \Omega) ;\langle\boldsymbol{v} \cdot \boldsymbol{n}, 1\rangle_{\Sigma_{j}}=0,1 \leq j \leq J\right\} .
$$

We now consider the variational problem:

Find $(\boldsymbol{\omega}, \boldsymbol{u}, p)$ in $H_{0}(\operatorname{curl}, \Omega) \times \mathbb{D}(\Omega) \times L_{0}^{2}(\Omega)$, such that

$$
\begin{aligned}
& \forall \boldsymbol{v} \in \mathbb{D}(\Omega), \quad a(\boldsymbol{\omega}, \boldsymbol{u} ; \boldsymbol{v})+b(\boldsymbol{v}, p)=\langle\boldsymbol{f}, \boldsymbol{v}\rangle, \\
& \forall q \in L_{0}^{2}(\Omega), \quad b(\boldsymbol{u}, q)=0, \\
& \forall \boldsymbol{\varphi} \in H_{0}(\operatorname{curl}, \Omega), \quad c(\boldsymbol{\omega}, \boldsymbol{u} ; \boldsymbol{\varphi})=0,
\end{aligned}
$$

where $\langle\cdot, \cdot\rangle$ denotes the duality pairing between $H_{0}(\operatorname{div}, \Omega)$ and its dual space. The bilinear forms $a(\cdot, \cdot ; \cdot), b(\cdot, \cdot)$ and $c(\cdot, \cdot ; \cdot)$ are defined by

$$
\begin{aligned}
& a(\boldsymbol{\omega}, \boldsymbol{u} ; \boldsymbol{v})=\nu \int_{\Omega}(\operatorname{curl} \boldsymbol{\omega})(\boldsymbol{x}) \cdot \boldsymbol{v}(\boldsymbol{x}) \mathrm{d} \boldsymbol{x}, \quad b(\boldsymbol{v}, q)=-\int_{\Omega}(\operatorname{div} \boldsymbol{v})(\boldsymbol{x}) q(\boldsymbol{x}) \mathrm{d} x, \\
& c(\boldsymbol{\omega}, \boldsymbol{u} ; \boldsymbol{\varphi})=\int_{\Omega} \boldsymbol{\omega}(\boldsymbol{x}) \cdot \boldsymbol{\varphi}(\boldsymbol{x}) \mathrm{d} \boldsymbol{x}-\int_{\Omega} \boldsymbol{u}(\boldsymbol{x}) \cdot(\boldsymbol{c u r l} \boldsymbol{\varphi})(\boldsymbol{x}) \mathrm{d} \boldsymbol{x} .
\end{aligned}
$$

To prove the equivalence of this problem with system (2.2)-(2.3), we need the following density results. Their proof can be found in [15], Chapter I, Section 2, for instance.

Lemma 2.2. The space of infinitely differentiable functions with a compact support in $\Omega$ and values in $\mathbb{R}^{d}$ is dense in $H_{0}(\operatorname{div}, \Omega)$. The space of infinitely differentiable functions with a compact support in $\Omega$ and values in $\mathbb{R}^{\frac{d(d-1)}{2}}$ is dense in $H_{0}(\mathbf{c u r l}, \Omega)$.

These results lead to the following statement. It involves the solutions $q_{j}^{t}, 1 \leq j \leq J$, of the problem (see [2], Prop. 3.14, for more details on these functions)

$$
\begin{cases}-\Delta q_{j}^{t}=0 & \text { in } \Omega^{\circ}, \\ \partial_{n} q_{j}^{t}=0 & \text { on } \partial \Omega, \\ {\left[q_{j}^{t}\right]_{j^{\prime}}=\text { constant },} & 1 \leq j^{\prime} \leq J, \\ {\left[\partial_{n} q_{j}^{t}\right]_{j^{\prime}}=0,} & 1 \leq j^{\prime} \leq J, \\ \left\langle\partial_{n} q_{j}^{t}, 1\right\rangle_{\Sigma_{j^{\prime}}}=\delta_{j j^{\prime}}, & 1 \leq j^{\prime} \leq J,\end{cases}
$$

where $[\cdot]_{j^{\prime}}$ denotes the jump through $\Sigma_{j^{\prime}}$ (making its sign precise is not needed in what follows). Note that each $\widetilde{\operatorname{grad}} q_{j}^{t}$ belongs to $H_{0}(\operatorname{div}, \Omega)$, where grad stands for the gradient defined in the distribution sense on $\Omega^{\circ}$, and that $H_{0}(\operatorname{div}, \Omega)$ is the direct sum of $\mathbb{D}(\Omega)$ and of the space spanned by the $\widetilde{\operatorname{grad}} q_{j}^{t}, 1 \leq j \leq J$.

Proposition 2.3. For any data $\boldsymbol{f}$ in the dual space of $H_{0}(\operatorname{div}, \Omega)$ satisfying

$$
\left\langle\boldsymbol{f}, \widetilde{\operatorname{grad}} q_{j}^{t}\right\rangle=0, \quad 1 \leq j \leq J,
$$

problems (2.2)-(2.3) and (2.9) are equivalent, in the sense that any triple $(\boldsymbol{\omega}, \boldsymbol{u}, p)$ in $H(\mathbf{c u r l}, \Omega) \times H(\operatorname{div}, \Omega) \times$ $L_{0}^{2}(\Omega)$ is a solution of problem (2.2)-(2.3) (with the first three equations of (2.2) satisfied in the distribution sense) if and only if it is a solution of problem (2.9).

Note that this statement does not hold for system (2.1)-(2.3), since the space of infinitely differentiable functions with a compact support in $\Omega$ and values in $\mathbb{R}^{d}$ is not dense in the space of functions of $H^{1}(\Omega)^{d}$ with 
zero normal trace. So formulation (2.2)-(2.3) seems more natural for handling the type of boundary conditions that we consider.

We briefly recall from [18], [4], Section 2, and [7], Section 2.5, the main arguments for proving the wellposedness of problem (2.9). It is readily checked that the kernel

$$
V=\left\{\boldsymbol{v} \in \mathbb{D}(\Omega) ; \forall q \in L_{0}^{2}(\Omega), b(\boldsymbol{v}, q)=0\right\},
$$

coincides with the space of divergence-free functions in $\mathbb{D}(\Omega)$. Similarly, the kernel

$$
\mathcal{W}=\left\{(\boldsymbol{\vartheta}, \boldsymbol{w}) \in H_{0}(\operatorname{curl}, \Omega) \times V ; \forall \boldsymbol{\varphi} \in H_{0}(\operatorname{curl}, \Omega), c(\boldsymbol{\vartheta}, \boldsymbol{w} ; \boldsymbol{\varphi})=0\right\},
$$

coincides with the space of pairs $(\boldsymbol{\vartheta}, \boldsymbol{w})$ in $H_{0}(\mathbf{c u r l}, \Omega) \times V$ such that $\boldsymbol{\vartheta}$ is equal to $\operatorname{curl} \boldsymbol{w}$ in the distribution sense. We observe that, for any solution $(\boldsymbol{\omega}, \boldsymbol{u}, p)$ of problem $(2.9)$, the pair $(\boldsymbol{\omega}, \boldsymbol{u})$ is a solution of the following reduced problem:

Find $(\boldsymbol{\omega}, \boldsymbol{u})$ in $\mathcal{W}$, such that

$$
\forall \boldsymbol{v} \in V, \quad a(\boldsymbol{\omega}, \boldsymbol{u} ; \boldsymbol{v})=\langle\boldsymbol{f}, \boldsymbol{v}\rangle .
$$

We recall from [4], Lemma 2.3, and [7], Propositions 2.5.3 and 2.5.4, the following properties (note that they require the further conditions on the $\Sigma_{j}$ which are enforced in the definition of $\mathbb{D}(\Omega)$ ).

Lemma 2.4. There exists a constant $\alpha>0$ such that

$$
\begin{aligned}
\forall \boldsymbol{v} \in V \backslash\{0\}, & \sup _{(\boldsymbol{\omega}, \boldsymbol{u}) \in \mathcal{W}} a(\boldsymbol{\omega}, \boldsymbol{u} ; \boldsymbol{v})>0, \\
& \forall(\boldsymbol{\omega}, \boldsymbol{u}) \in \mathcal{W}, \quad \sup _{\boldsymbol{v} \in V} \frac{a(\boldsymbol{\omega}, \boldsymbol{u} ; \boldsymbol{v})}{\|\boldsymbol{v}\|_{L^{2}(\Omega)^{d}}} \geq \alpha\left(\|\boldsymbol{\omega}\|_{H(\operatorname{curl}, \Omega)}+\|\boldsymbol{u}\|_{L^{2}(\Omega)^{d}}\right) .
\end{aligned}
$$

When combining these properties with [15], Chapter I, Lemma 4.1, we derive that problem (2.15) has a unique solution $(\boldsymbol{\omega}, \boldsymbol{u})$ in $\mathcal{W}$. We also recall the standard inf-sup condition on the form $b(\cdot, \cdot)$, see [15], Chapter I, Corollary 2.4, for instance.

Lemma 2.5. There exists a constant $\beta>0$ such that

$$
\forall q \in L_{0}^{2}(\Omega), \quad \sup _{\boldsymbol{v} \in H_{0}(\operatorname{div}, \Omega)} \frac{b(\boldsymbol{v}, q)}{\|\boldsymbol{v}\|_{H(\operatorname{div}, \Omega)}} \geq \beta\|q\|_{L^{2}(\Omega)} .
$$

When applying this result with $\Omega$ replaced by $\Omega^{\circ}$, we easily derive that

$$
\forall q \in L_{0}^{2}(\Omega), \quad \sup _{\boldsymbol{v} \in \mathbb{D}(\Omega)} \frac{b(\boldsymbol{v}, q)}{\|\boldsymbol{v}\|_{H(\operatorname{div}, \Omega)}} \geq \beta\|q\|_{L^{2}(\Omega)} .
$$

Combining this with (2.16) and applying the arguments in [7], Theorem 1.3.11 yield the well-posedness of problem (2.9).

Theorem 2.6. For any data $\boldsymbol{f}$ in the dual space of $H_{0}(\operatorname{div}, \Omega)$, problem (2.9) has a unique solution $(\boldsymbol{\omega}, \boldsymbol{u}, p)$ in $H_{0}(\operatorname{curl}, \Omega) \times \mathbb{D}(\Omega) \times L_{0}^{2}(\Omega)$. Moreover this solution satisfies

$$
\|\boldsymbol{\omega}\|_{H(\operatorname{curl}, \Omega)}+\|\boldsymbol{u}\|_{H(\operatorname{div}, \Omega)}+\|p\|_{L^{2}(\Omega)} \leq c\|\boldsymbol{f}\|_{H_{0}(\operatorname{div}, \Omega)^{\prime}} .
$$

We conclude with some regularity properties of the solution of problem (2.9) which can easily be derived from [2], Section 2, [11] and [12] both in the two- and three-dimensional cases: 
- In dimension $d=2$, the mapping: $\boldsymbol{f} \mapsto(\boldsymbol{\omega}, \boldsymbol{u}, p)$, where $(\boldsymbol{\omega}, \boldsymbol{u}, p)$ is the solution of problem (2.9) with data $\boldsymbol{f}$, is continuous from $H^{\max \{0, s-1\}}(\Omega)^{d}$ into $H^{s}(\Omega)^{\frac{d(d-1)}{2}} \times H^{s}(\Omega)^{d} \times H^{s}(\Omega)$, for

(i) all $s \leq \frac{1}{2}$ in the general case;

(ii) all $s \leq 1$ when $\Omega$ is convex;

(iii) all $s<\frac{\pi}{\alpha}$ when $\Omega$ is a polygon with largest angle equal to $\alpha$.

Moreover, when the data $f$ belongs to $L^{2}(\Omega)^{d}$, both the pressure $p$ and the vorticity $\omega$ belong to $H^{1}(\Omega)$.

- In dimension $d=3$, the mapping: $\boldsymbol{f} \mapsto(\boldsymbol{\omega}, \boldsymbol{u}, p)$, where $(\boldsymbol{\omega}, \boldsymbol{u}, p)$ is the solution of problem $(2.9)$ with data $\boldsymbol{f}$, is continuous from $H^{\max \{0, s-1\}}(\Omega)^{d}$ into $H^{s}(\Omega)^{\frac{d(d-1)}{2}} \times H^{s}(\Omega)^{d} \times H^{s}(\Omega)$, for

(i) all $s \leq \frac{1}{2}$ in the general case;

(ii) all $s \leq 1$ when $\Omega$ is convex.

\section{The SPeCtral Element Discrete PROBlem}

From now on, we assume that $\Omega$ admits a partition without overlap into a finite number of subdomains

$$
\bar{\Omega}=\cup_{k=1}^{K} \Omega_{k} \quad \text { and } \quad \Omega_{k} \cap \Omega_{k^{\prime}}=\emptyset, \quad 1 \leq k<k^{\prime} \leq K,
$$

which satisfy the further conditions:

(i) Each $\Omega_{k}, 1 \leq k \leq K$, is a rectangle in dimension $d=2$ or a rectangular parallelepiped in dimension $d=3$;

(ii) The intersection of two subdomains $\bar{\Omega}_{k}$ and $\bar{\Omega}_{k^{\prime}}, 1 \leq k<k^{\prime} \leq K$, if not empty, is either a vertex or a whole edge or a whole face of both $\Omega_{k}$ and $\Omega_{k^{\prime}}$;

(iii) The $\bar{\Sigma}_{j}, 1 \leq j \leq J$, introduced in Notation 2.1, are the union of whole edges $(d=2)$ or faces $(d=3)$ of some $\Omega_{k}$.

The discrete spaces are constructed from the finite elements proposed by Nédélec on cubic three-dimensional meshes, see [16], Section 2. In order to describe them and for any triple $(\ell, m, n)$ of nonnegative integers, we introduce

- in dimension $d=2$, the space $\mathbb{P}_{\ell, m}\left(\Omega_{k}\right)$ of restrictions to $\Omega_{k}$ of polynomials with degree $\leq \ell$ with respect to $x$ and $\leq m$ with respect to $y$;

- in dimension $d=3$, the space $\mathbb{P}_{\ell, m, n}\left(\Omega_{k}\right)$ of restrictions to $\Omega_{k}$ of polynomials with degree $\leq \ell$ with respect to $x, \leq m$ with respect to $y$ and $\leq n$ with respect to $z$.

When $\ell$ and $m$ are equal to $n$, these spaces are simply denoted by $\mathbb{P}_{n}\left(\Omega_{k}\right)$. Relying on these definitions, we introduce the local spaces, for an integer $N \geq 2$,

$$
\begin{gathered}
D_{N}^{k}= \begin{cases}\mathbb{P}_{N, N-1}\left(\Omega_{k}\right) \times \mathbb{P}_{N-1, N}\left(\Omega_{k}\right) & \text { if } d=2, \\
\mathbb{P}_{N, N-1, N-1}\left(\Omega_{k}\right) \times \mathbb{P}_{N-1, N, N-1}\left(\Omega_{k}\right) \times \mathbb{P}_{N-1, N-1, N}\left(\Omega_{k}\right) & \text { if } d=3,\end{cases} \\
C_{N}^{k}=\left\{\begin{array}{l}
\mathbb{P}_{N}\left(\Omega_{k}\right) \\
\mathbb{P}_{N-1, N, N}\left(\Omega_{k}\right) \times \mathbb{P}_{N, N-1, N}\left(\Omega_{k}\right) \times \mathbb{P}_{N, N, N-1}\left(\Omega_{k}\right) \\
\text { if } d=2,
\end{array}\right. \\
M_{N}^{k}=\mathbb{P}_{N-1}\left(\Omega_{k}\right) .
\end{gathered}
$$

The space $\mathbb{D}_{N}$ which approximates $H_{0}(\operatorname{div}, \Omega)$ is then defined by

$$
\mathbb{D}_{N}=\left\{\boldsymbol{v}_{N} \in \mathbb{D}(\Omega) ;\left.\boldsymbol{v}_{N}\right|_{\Omega_{k}} \in D_{N}^{k}, 1 \leq k \leq K\right\}
$$

The space $\mathbb{C}_{N}$ which approximates $H_{0}(\mathbf{c u r l}, \Omega)$ is defined by

$$
\mathbb{C}_{N}=\left\{\boldsymbol{\varphi}_{N} \in H_{0}(\operatorname{curl}, \Omega) ;\left.\boldsymbol{\varphi}_{N}\right|_{\Omega_{k}} \in C_{N}^{k}, 1 \leq k \leq K\right\} .
$$


Finally, for the approximation of $L_{0}^{2}(\Omega)$, we consider the space

$$
\mathbb{M}_{N}=\left\{q_{N} \in L_{0}^{2}(\Omega) ;\left.q_{N}\right|_{\Omega_{k}} \in M_{N}^{k}, 1 \leq k \leq K\right\}
$$

It can be noted that the functions in $\mathbb{D}_{N}$ have continuous normal traces through the interfaces $\bar{\Omega}_{k} \cap \bar{\Omega}_{k^{\prime}}$ while the functions in $\mathbb{C}_{N}$ have continuous traces in dimension $d=2$, continuous tangential traces in dimension $d=3$. Thanks to the previous choice, the discretization that we propose is perfectly conforming.

Setting $\xi_{0}=-1$ and $\xi_{N}=1$, we introduce the $N-1$ nodes $\xi_{j}, 1 \leq j \leq N-1$, and the $N+1$ weights $\rho_{j}$, $0 \leq j \leq N$, of the Gauss-Lobatto quadrature formula on $[-1,1]$. Denoting by $\mathbb{P}_{n}(-1,1)$ the space of restrictions to $[-1,1]$ of polynomials with degree $\leq n$, we recall that the following equality holds

$$
\forall \Phi \in \mathbb{P}_{2 N-1}(-1,1), \quad \int_{-1}^{1} \Phi(\zeta) \mathrm{d} \zeta=\sum_{j=0}^{N} \Phi\left(\xi_{j}\right) \rho_{j}
$$

We also recall from [5], formula (13.20), the following property, which is useful in what follows

$$
\forall \varphi_{N} \in \mathbb{P}_{N}(-1,1), \quad\left\|\varphi_{N}\right\|_{L^{2}(-1,1)}^{2} \leq \sum_{j=0}^{N} \varphi_{N}^{2}\left(\xi_{j}\right) \rho_{j} \leq 3\left\|\varphi_{N}\right\|_{L^{2}(-1,1)}^{2}
$$

Denoting by $F_{k}$ the affine mapping that sends $]-1,1\left[{ }^{d}\right.$ onto $\Omega_{k}$, we introduce the local discrete products, defined on continuous functions $u$ and $v$ on $\bar{\Omega}_{k}$ by

$$
(u, v)_{N}^{k}= \begin{cases}\frac{\operatorname{meas}\left(\Omega_{k}\right)}{4} \sum_{i=0}^{N} \sum_{j=0}^{N} u \circ F_{k}\left(\xi_{i}, \xi_{j}\right) v \circ F_{k}\left(\xi_{i}, \xi_{j}\right) \rho_{i} \rho_{j} & \text { if } \quad d=2, \\ \frac{\operatorname{meas}\left(\Omega_{k}\right)}{8} \sum_{i=0}^{N} \sum_{j=0}^{N} \sum_{p=0}^{N} u \circ F_{k}\left(\xi_{i}, \xi_{j}, \xi_{p}\right) v \circ F_{k}\left(\xi_{i}, \xi_{j}, \xi_{p}\right) \rho_{i} \rho_{j} \rho_{p} & \text { if } \quad d=3 .\end{cases}
$$

The global product is then defined on continuous functions $u$ and $v$ on $\bar{\Omega}$ by

$$
((u, v))_{N}=\sum_{k=1}^{K}\left(\left.u\right|_{\Omega_{k}},\left.v\right|_{\Omega_{k}}\right)_{N}^{k}
$$

The discrete problem is now constructed from (2.9) by using the Galerkin method combined with numerical integration. It reads:

Find $\left(\boldsymbol{\omega}_{N}, \boldsymbol{u}_{N}, p_{N}\right)$ in $\mathbb{C}_{N} \times \mathbb{D}_{N} \times \mathbb{M}_{N}$, such that

$$
\begin{array}{ll}
\forall \boldsymbol{v}_{N} \in \mathbb{D}_{N}, & a_{N}\left(\boldsymbol{\omega}_{N}, \boldsymbol{u}_{N} ; \boldsymbol{v}_{N}\right)+b_{N}\left(\boldsymbol{v}_{N}, p_{N}\right)=\left(\left(\boldsymbol{f}, \boldsymbol{v}_{N}\right)\right)_{N}, \\
\forall q_{N} \in \mathbb{M}_{N}, \quad b_{N}\left(\boldsymbol{u}_{N}, q_{N}\right)=0, \\
\forall \boldsymbol{\varphi}_{N} \in \mathbb{C}_{N}, \quad c_{N}\left(\boldsymbol{\omega}_{N}, \boldsymbol{u}_{N} ; \boldsymbol{\varphi}_{N}\right)=0,
\end{array}
$$

where the bilinear forms $a_{N}(\cdot, \cdot ; \cdot), b_{N}(\cdot, \cdot)$ and $c_{N}(\cdot, \cdot ; \cdot)$ are defined by

$$
\begin{array}{r}
a_{N}\left(\boldsymbol{\omega}_{N}, \boldsymbol{u}_{N} ; \boldsymbol{v}_{N}\right)=\nu\left(\left(\operatorname{curl} \boldsymbol{\omega}_{N}, \boldsymbol{v}_{N}\right)\right)_{N}, \quad b_{N}\left(\boldsymbol{v}_{N}, q_{N}\right)=-\left(\left(\operatorname{div} \boldsymbol{v}_{N}, q_{N}\right)\right)_{N}, \\
c_{N}\left(\boldsymbol{\omega}_{N}, \boldsymbol{u}_{N} ; \boldsymbol{\varphi}_{N}\right)=\left(\left(\boldsymbol{\omega}_{N}, \boldsymbol{\varphi}_{N}\right)\right)_{N}-\left(\left(\boldsymbol{u}_{N}, \operatorname{curl} \boldsymbol{\varphi}_{N}\right)\right)_{N} .
\end{array}
$$

It follows from (3.7) combined with Cauchy-Schwarz inequalities that the forms $a_{N}(\cdot, \cdot ; \cdot), b_{N}(\cdot, \cdot)$ and $c_{N}(\cdot, \cdot ; \cdot)$ are continuous on $\left(\mathbb{C}_{N} \times \mathbb{D}_{N}\right) \times \mathbb{D}_{N}, \mathbb{D}_{N} \times \mathbb{M}_{N}$ and $\left(\mathbb{C}_{N} \times \mathbb{D}_{N}\right) \times \mathbb{C}_{N}$, respectively, with norms bounded independently of $N$. Moreover, as a consequence of the exactness property $(3.6)$, the forms $b(\cdot, \cdot)$ and $b_{N}(\cdot, \cdot)$ coincide on $\mathbb{D}_{N} \times \mathbb{M}_{N}$. 
In order to perform the numerical analysis of problem (3.10), we first recall from the finite element analogous result [16] that the range of $\mathbb{D}_{N}$ by the divergence operator is contained in $\mathbb{M}_{N}$. So, if $V_{N}$ denotes the kernel

$$
V_{N}=\left\{\boldsymbol{v}_{N} \in \mathbb{D}_{N} ; \forall q_{N} \in \mathbb{M}_{N}, b_{N}\left(\boldsymbol{v}_{N}, q_{N}\right)=0\right\},
$$

it is readily checked by taking $q_{N}$ equal to $\operatorname{div} \boldsymbol{v}_{N}$ in the previous line that $V_{N}$ is the space of divergence-free functions in $\mathbb{D}_{N}$, i.e. coincides with $\mathbb{D}_{N} \cap V$.

We now investigate some properties of the curl operator. It follows from [16] (see also [10], Thm. 2.1) that the range of $\mathbb{C}_{N}$ by the curl operator is contained in $\mathbb{D}_{N}$. We also have the following result, which requires some further notation.

Notation 3.1. Let $\Gamma_{i}, 0 \leq i \leq I$, be the connected components of $\partial \Omega$ such that $\Gamma_{0}$ is the boundary of the only unbounded connected component of $\mathbb{R}^{3} \backslash \bar{\Omega}$.

We can now define the space

$$
H_{\diamond}^{1}(\Omega)=\left\{\mu \in H^{1}(\Omega) ; \mu=0 \text { on } \Gamma_{0} \text { and } \mu=\operatorname{constant} \text { on } \Gamma_{i}, 1 \leq i \leq I\right\} .
$$

Lemma 3.2. The kernel of the curl operator in $\mathbb{C}_{N}$ is reduced to $\{0\}$ in dimension $d=2$, equal to the range of the space $\mathbb{G}_{N}$ by the gradient operator in dimension $d=3$, where $\mathbb{G}_{N}$ denotes the space

$$
\mathbb{G}_{N}=\left\{\mu_{N} \in H_{\diamond}^{1}(\Omega) ;\left.\mu_{N}\right|_{\Omega_{k}} \in \mathbb{P}_{N}\left(\Omega_{k}\right), 1 \leq k \leq K\right\} .
$$

Proof. In dimension $d=2$, a curl-free function $\varphi_{N}$ in $\mathbb{C}_{N}$ is constant on $\Omega$. Since it vanishes on $\partial \Omega$, it is zero. In dimension $d=3$, let $\varphi_{N}$ be a curl-free function in $\mathbb{C}_{N}$. Then using [15], Chapter I, Theorem 2.9, yields that, since the domain $\Omega^{\circ}$ introduced in Notation 2.1 is simply connected, $\varphi_{N}$ is equal on $\Omega^{\circ}$ to the gradient of a function $\mu$ in $H^{1}\left(\Omega^{\circ}\right)$, which is defined up to an additive constant. The identity $\varphi_{N}=\operatorname{grad} \mu$ on $\Omega_{k}$ yields that each $\left.\mu\right|_{\Omega_{k}}$ belongs to $\mathbb{P}_{N}\left(\Omega_{k}\right)$. Finally, it follows from the fact that $\gamma_{t}\left(\boldsymbol{\varphi}_{N}\right)$ vanishes on $\partial \Omega$, that $\mu$ has a zero tangential gradient on $\partial \Omega$, hence is constant on each $\Gamma_{i}$. It also follows from the fact that $\gamma_{t}\left(\boldsymbol{\varphi}_{N}\right)$ is continuous through each $\Sigma_{j}$ that the tangential gradient of the jump of $\mu$ through each $\Sigma_{j}$ is zero, so that the jump of $\mu$ is constant. Since $\mu$ is constant on each $\Gamma_{i}$, the jump of $\mu$ through each $\bar{\Sigma}_{j} \cap \Gamma_{i}$ is zero, hence the jump of $\mu$ through each $\Sigma_{j}$ is zero. Thus, $\mu$ belongs to $H^{1}(\Omega)$. Finally, subtracting to $\mu$ its value on $\Gamma_{0}$ yields that $\varphi_{N}$ is the gradient of a function in $\mathbb{G}_{N}$. Conversely, it is readily checked that the gradients of all functions in $\mathbb{G}_{N}$ belong to $\mathbb{C}_{N}$ and are curl-free.

We are now in a position to state and prove the key result of this section.

Proposition 3.3. There exists an operator $A_{N}$ from $V_{N}$ into $\mathbb{C}_{N}$

(i) which satisfies

$$
\forall \boldsymbol{v}_{N} \in V_{N}, \quad \operatorname{curl} A_{N}\left(\boldsymbol{v}_{N}\right)=\boldsymbol{v}_{N} ;
$$

(ii) such that, in dimension $d=3$,

$$
\forall \mu_{N} \in \mathbb{G}_{N}, \quad\left(\left(A_{N}\left(\boldsymbol{v}_{N}\right), \operatorname{grad} \mu_{N}\right)\right)_{N}=0 ;
$$

(iii) which satisfies, for a constant $c$ independent of $N$,

$$
\forall \boldsymbol{v}_{N} \in V_{N}, \quad\left\|A_{N}\left(\boldsymbol{v}_{N}\right)\right\|_{H(\operatorname{curl}, \Omega)} \leq c\left\|\boldsymbol{v}_{N}\right\|_{L^{2}(\Omega)^{d}} .
$$

Note from Lemma 3.2 that this operator is uniquely defined by (3.14) and the further condition (3.15) in dimension $d=3$. The proof of this proposition is rather technical, so that we prefer to give it separetely in dimensions $d=2$ and $d=3$. 
Proof. Case of dimension $d=2$

Let $\boldsymbol{v}_{N}$ be any polynomial in $V_{N}$. We assume that $\Omega$ is contained in a rectangle $\left.\Omega^{*}=\right] a, a^{\prime}[\times] b, b^{\prime}[$, and we denote by $\overline{\boldsymbol{v}}_{N}$ the extension of $\boldsymbol{v}_{N}$ by zero to $\Omega^{*}$. So, $\overline{\boldsymbol{v}}_{N}$ is still divergence-free on $\Omega^{*}$. Denoting its components by $\bar{v}_{N x}$ and $\bar{v}_{N y}$, we consider the function defined on $\Omega^{*}$ by

$$
\psi_{N}(x, y)=\int_{b}^{y} \bar{v}_{N x}(x, \eta) \mathrm{d} \eta
$$

It is readily checked that each $\left.\psi_{N}\right|_{\Omega_{k}}$ belongs to $\mathbb{P}_{N}\left(\Omega_{k}\right)$. The continuity of $\psi_{N}$ through each horizontal edge shared by two subdomains $\Omega_{k}$ (where horizontal edge means an edge contained in a line $y=y_{0}$ ) follows from its definition. Moreover, since $\bar{v}_{N x}=\overline{\boldsymbol{v}}_{N} \cdot \boldsymbol{n}$ is continuous through all vertical edges shared by two subdomains $\Omega_{k}$, the same property holds for $\psi_{N}$. So it belongs to $H(\operatorname{curl}, \Omega)$. On the other hand, we observe that, since $\overline{\boldsymbol{v}}_{N}$ is divergence-free,

$$
\left.\left(\partial_{x} \psi_{N}\right)\right|_{\Omega_{k}}(x, y)=\int_{b}^{y}\left(\partial_{x} \bar{v}_{N x}\right)(x, \eta) \mathrm{d} \eta=-\int_{b}^{y}\left(\partial_{y} \bar{v}_{N y}\right)(x, \eta) \mathrm{d} \eta=-\bar{v}_{N y}(x, y) .
$$

This equation yields that $\operatorname{curl} \psi_{N}$ is equal to $\boldsymbol{v}_{N}$ on $\Omega$. Finally, since

- $\partial_{x} \psi_{N}$ vanishes on the horizontal edges of $\Omega$ and on $\Omega^{*} \backslash \bar{\Omega}$;

- $\partial_{y} \psi_{N}$ vanishes on the vertical edges of $\Omega$ and also on $\Omega^{*} \backslash \bar{\Omega}$;

- and $\psi_{N}$ is zero at $(a, b)$;

it is zero on $\Gamma_{0}$ and equal to a constant $c_{i}$ on each $\Gamma_{i}, 1 \leq i \leq I$. Then, it follows from the condition $\left\langle\boldsymbol{v}_{N} \cdot \boldsymbol{n}, 1\right\rangle_{\Sigma_{j}}=0$ that all these constants are equal to zero. So, $\psi_{N}$ belongs to $\mathbb{C}_{N}$ and satisfies $\operatorname{curl} \psi_{N}=\boldsymbol{v}_{N}$ on $\Omega$. From Lemma 3.2, the restriction of this $\psi_{N}$ to $\Omega$ thus coincides with $A_{N}\left(\boldsymbol{v}_{N}\right)$. Moreover estimate (3.16) follows from a simple Poincaré-Friedrichs inequality applied to (3.17).

Proof. Case of dimension $d=3$

The construction of a function $\boldsymbol{\psi}_{N}$ is now performed in four steps.

1) Like in dimension $d=2$, we assume that $\Omega$ is contained in a rectangular parallelepiped $\left.\Omega^{*}=\right] a, a^{\prime}[\times] b, b^{\prime}[\times] c, c^{\prime}[$, and we denote by $\overline{\boldsymbol{v}}_{N}$ the extension of $\boldsymbol{v}_{N}$ by zero to $\Omega^{*}$. Denoting its components by $\bar{v}_{N x}, \bar{v}_{N y}$ and $\bar{v}_{N z}$, we first define a function $\boldsymbol{\psi}_{N}^{\sharp}=\left(\psi_{N x}^{\sharp}, \psi_{N y}^{\sharp}, \psi_{N z}^{\sharp}\right)$ by

$$
\psi_{N x}^{\sharp}(x, y, z)=\int_{c}^{z} \bar{v}_{N y}(x, y, \zeta) \mathrm{d} \zeta, \quad \psi_{N y}^{\sharp}(x, y, z)=-\int_{c}^{z} \bar{v}_{N x}(x, y, \zeta) \mathrm{d} \zeta, \quad \psi_{N z}^{\sharp}=0 .
$$

The first two components of $\left.\boldsymbol{\psi}_{N}^{\sharp}\right|_{\Omega_{k}}$ belong to $\mathbb{P}_{N-1, N, N}\left(\Omega_{k}\right)$ and $\mathbb{P}_{N, N-1, N}\left(\Omega_{k}\right)$, respectively, so that $\left.\boldsymbol{\psi}_{N}^{\sharp}\right|_{\Omega_{k}}$ belongs to $C_{N}^{k}$. This function is such that the first two components of its curl are equal to $\bar{v}_{N x}$ and $\bar{v}_{N y}$. Moreover, since $\boldsymbol{v}_{N}$ belongs to $V_{N}, \overline{\boldsymbol{v}}_{N}$ is divergence-free. This yields

$$
\left(\partial_{x} \psi_{N y}^{\sharp}-\partial_{y} \psi_{N x}^{\sharp}\right)(x, y, z)=-\int_{c}^{z}\left(\partial_{x} \bar{v}_{N x}+\partial_{y} \bar{v}_{N y}\right)(x, y, \zeta) \mathrm{d} \zeta=\int_{c}^{z}\left(\partial_{z} \bar{v}_{N z}\right)(x, y, \zeta) \mathrm{d} \zeta=v_{N z}(x, y, z) .
$$

So, $\operatorname{curl} \boldsymbol{\psi}_{N}^{\sharp}$ is equal to $\boldsymbol{v}_{N}$ on each $\Omega_{k}$. Moreover the continuity of $\psi_{N x}^{\sharp}$ through each face of two $\Omega_{k}$ contained in a plane $y=y_{0}$ and $z=z_{0}$ follows from its definition and the property of $\boldsymbol{v}_{N}$. Simillarly, $\psi_{N y}^{\sharp}$ is continuous through each face of two $\Omega_{k}$ contained in a plane $x=x_{0}$ and $z=z_{0}$, so that $\boldsymbol{\psi}_{N}^{\sharp}$ belongs to $H(\mathbf{c u r l}, \Omega)$. Moreover the following inequality is easily derived from (3.18)

$$
\left\|\boldsymbol{\psi}_{N}^{\sharp}\right\|_{H(\operatorname{curl}, \Omega)} \leq c\left\|\boldsymbol{v}_{N}\right\|_{L^{2}(\Omega)^{3}} .
$$


2) Noting that $\partial \Omega$ is contained in the union of a finite number of planes, we denote by $\gamma_{\ell}, 1 \leq \ell \leq L$, the connected components of the intersections of $\partial \Omega$ with these planes. For each $\gamma_{\ell}$, according as $\gamma_{\ell}$ is contained in a plane $x=x_{0}$ or in a plane $y=y_{0}$ or in a plane $z=z_{0}$, we set

$$
\begin{aligned}
& g_{N y}^{\ell}(y, z)=-\int_{c}^{z} \bar{v}_{N x}\left(x_{0}, y, \zeta\right) \mathrm{d} \zeta, \quad g_{N z}^{\ell}(y, z)=0 \\
& \text { or } \quad g_{N x}^{\ell}(x, z)=\int_{c}^{z} \bar{v}_{N y}\left(x, y_{0}, \zeta\right) \mathrm{d} \zeta, \quad g_{N z}^{\ell}(x, z)=0 \\
& \quad \text { or } \quad g_{N x}^{\ell}(x, y)=\int_{c}^{z_{0}} \bar{v}_{N y}(x, y, \zeta) \mathrm{d} \zeta, \quad g_{N y}^{\ell}(x, y)=-\int_{c}^{z_{0}} \bar{v}_{N x}(x, y, \zeta) \mathrm{d} \zeta .
\end{aligned}
$$

We observe that the vector $\boldsymbol{g}_{N}^{\ell}$ with these components is tangential to $\gamma_{\ell}$ and that its restriction to each intersection $\gamma_{\ell} \cap \partial \Omega_{k}$ which has a positive measure in $\gamma_{\ell}$ belongs to $\mathbb{P}_{N-1, N}\left(\gamma_{\ell} \cap \partial \Omega_{k}\right) \times \mathbb{P}_{N, N-1}\left(\gamma_{\ell} \cap \partial \Omega_{k}\right)$, with obvious notation for these new spaces. Moreover, the two-dimensional curl of these functions $\boldsymbol{g}_{N}^{\ell}$ is equal to zero on each $\gamma_{\ell}$ (indeed, $\partial_{z} g_{N y}^{\ell}$ vanishes on the faces contained in a plane $x=x_{0}, \partial_{z} g_{N x}^{\ell}$ vanishes on the faces contained in a plane $y=y_{0}$ and $\left(\partial_{x} g_{N y}^{\ell}-\partial_{y} g_{N x}^{\ell}\right)(x, y)=\boldsymbol{v}_{N z}\left(x, y, z_{0}\right)$ also vanishes on the faces contained in a plane $z=z_{0}$ ) and the tangential components of $\boldsymbol{g}_{N}^{\ell}$ and $\boldsymbol{g}_{N}^{\ell^{\prime}}$ on each edge shared by $\gamma_{\ell}$ and $\gamma_{\ell^{\prime}}$ are equal. Since $\partial \Omega \backslash \cup_{j=1}^{N} \partial \Sigma_{j}$ is simply-connected, it follows from [9], Proposition 3.1, that there exists a function $k_{N}$ in $H^{1}\left(\partial \Omega \backslash \cup_{j=1}^{N} \partial \Sigma_{j}\right)$, vanishing at a corner of $\Gamma_{0}$, such that the tangential gradient of the restriction of $k_{N}$ to each $\gamma_{\ell}$ is equal to $\boldsymbol{g}_{N}^{\ell}$. Moreover the following estimate can be derived from [9], Proposition 4.7 (a more complete proof of it would involve complex notation, so that we have rather avoid it and refer to [9] for the details)

$$
\left\|k_{N}\right\|_{H^{\frac{1}{2}}\left(\partial \Omega \backslash \cup_{j=1}^{N} \partial \Sigma_{j}\right)} \leq c\left\|\boldsymbol{\psi}_{N}^{\sharp} \times \boldsymbol{n}\right\|_{H^{-\frac{1}{2}}(\partial \Omega)} .
$$

Note that the restriction of $k_{N}$ to each $\gamma_{\ell} \cap \bar{\Omega}_{k}$ which has a positive measure in $\gamma_{\ell}$ belongs to $\mathbb{P}_{N}\left(\gamma_{\ell} \cap \bar{\Omega}_{k}\right)$ and that the jump of $k_{N}$ through each $\partial \Sigma_{j}$ is constant.

3) We recall from [6], Chapter II, Theorem 4.1, that, if $\gamma$ denotes a face of $\Omega_{k}$ that is contained in $\partial \Omega$, there exists a lifting operator $\mathcal{L}_{k}^{\gamma}$ from $\mathbb{P}_{N}(\gamma)$ into $\mathbb{P}_{N}\left(\Omega_{k}\right)$ such that, for any $\varphi_{N}$ in $\mathbb{P}_{N}(\gamma)$, the trace of $\mathcal{L}_{k}^{\gamma} \varphi_{N}$ is equal

- to $\varphi_{N}$ on $\gamma$;

- to zero on the opposite face to $\gamma$;

- and, when $\varphi_{N}$ is zero on an edge of $\gamma$, to zero on the face that shares this edge with $\gamma$.

We use iteratively this operator on the $\Omega_{k}, k=1, \ldots, K$, and on the faces $\gamma$ of $\Omega_{k}$ which are contained in $\partial \Omega$ and, at each step, we subtract from $k_{N}$ the trace of the new function to the other $\Omega_{k^{\prime}}, k^{\prime}>k$, that share a face or an edge with $\Omega_{k}$ (we refer to [6], Chap. II, for details on this procedure). Thus we derive the existence of a $\mu_{N}$ in $H^{1}\left(\Omega^{\circ}\right)$ such that $\boldsymbol{\psi}_{N}^{\sharp}-\widetilde{\operatorname{grad}} \mu_{N}$ belongs to $\mathbb{C}_{N}$ (we recall that $\widetilde{\text { grad }}$ denotes the gradient on $\Omega^{\circ}$ ). Moreover, it follows from [6], Chapter II, Theorem 4.1, that this function satisfies

$$
\left\|\widetilde{\operatorname{grad}} \mu_{N}\right\|_{L^{2}(\Omega)^{3}} \leq c\left\|k_{N}\right\|_{H^{\frac{1}{2}}\left(\partial \Omega \backslash \cup_{j=1}^{N} \partial \Sigma_{j}\right)},
$$

whence, thanks to (3.19) and (3.20),

$$
\widetilde{\operatorname{grad}} \mu_{N}\left\|_{L^{2}(\Omega)^{3}} \leq c^{\prime}\right\| \boldsymbol{v}_{N} \|_{L^{2}(\Omega)^{3}}
$$

4) Finally, the Lax-Milgram lemma combined with (3.7) and a generalized Poincaré-Friedrichs inequality yields that there exists a unique $\tilde{\mu}_{N}$ in $\mathbb{G}_{N}$ such that

$$
\forall \rho_{N} \in \mathbb{G}_{N}, \quad\left(\left(\operatorname{grad} \tilde{\mu}_{N}, \operatorname{grad} \rho_{N}\right)\right)_{N}=\left(\left(\psi_{N}^{\sharp}-\widetilde{\operatorname{grad}} \mu_{N}, \operatorname{grad} \rho_{N}\right)\right)_{N} .
$$


Moreover this function satisfies

$$
\left\|\operatorname{grad} \tilde{\mu}_{N}\right\|_{L^{2}(\Omega)^{3}} \leq 3^{\frac{3}{2}}\left(\left\|\boldsymbol{\psi}_{N}^{\sharp}\right\|_{L^{2}(\Omega)^{3}}+\left\|\widetilde{\operatorname{grad}} \mu_{N}\right\|_{L^{2}(\Omega)^{3}}\right) .
$$

The choice of $\tilde{\mu}_{N}$ yields that the function $\boldsymbol{\psi}_{N}=\boldsymbol{\psi}_{N}^{\sharp}-\widetilde{\operatorname{grad}} \mu_{N}-\operatorname{grad} \tilde{\mu}_{N}$ is equal to $A_{N}\left(\boldsymbol{v}_{N}\right)$, so that the desired estimate follows from (3.19), (3.21) and (3.22).

In analogy with the continuous case, we now introduce the discrete kernel

$$
\mathcal{W}_{N}=\left\{\left(\boldsymbol{\vartheta}_{N}, \boldsymbol{w}_{N}\right) \in \mathbb{C}_{N} \times V_{N} ; \forall \boldsymbol{\varphi}_{N} \in \mathbb{C}_{N}, c_{N}\left(\boldsymbol{\vartheta}_{N}, \boldsymbol{w}_{N} ; \boldsymbol{\varphi}_{N}\right)=0\right\},
$$

and observe that, for any solution $\left(\boldsymbol{\omega}_{N}, \boldsymbol{u}_{N}, p_{N}\right)$ of problem $(3.10)$, the pair $\left(\boldsymbol{\omega}_{N}, \boldsymbol{u}_{N}\right)$ is a solution of the reduced problem:

Find $\left(\boldsymbol{\omega}_{N}, \boldsymbol{u}_{N}\right)$ in $\mathcal{W}_{N}$, such that

$$
\forall \boldsymbol{v}_{N} \in V_{N}, \quad a_{N}\left(\boldsymbol{\omega}_{N}, \boldsymbol{u}_{N} ; \boldsymbol{v}_{N}\right)=\left(\left(\boldsymbol{f}, \boldsymbol{v}_{N}\right)\right)_{N}
$$

Thanks to Proposition 3.3, we are now in a position to prove the well-posedness of this problem.

Lemma 3.4. The form $a_{N}(\cdot, \cdot ; \cdot)$ satisfies the positivity property

$$
\forall \boldsymbol{v}_{N} \in V_{N} \backslash\{0\}, \quad \sup _{\left(\boldsymbol{\omega}_{N}, \boldsymbol{u}_{N}\right) \in \mathcal{W}_{N}} a_{N}\left(\boldsymbol{\omega}_{N}, \boldsymbol{u}_{N} ; \boldsymbol{v}_{N}\right)>0 .
$$

Proof. Let $\boldsymbol{v}_{N}$ be a polynomial in $V_{N}$ such that $a_{N}\left(\boldsymbol{\omega}_{N}, \boldsymbol{u}_{N} ; \boldsymbol{v}_{N}\right)$ vanishes for all pairs $\left(\boldsymbol{\omega}_{N}, \boldsymbol{u}_{N}\right)$ in $\mathcal{W}_{N}$. We set $\boldsymbol{\vartheta}_{N}=A_{N}\left(\boldsymbol{v}_{N}\right)$ and we consider the equation:

Find $\boldsymbol{z}_{N}$ in $V_{N}$, such that

$$
\forall \boldsymbol{w}_{N} \in V_{N}, \quad\left(\left(\boldsymbol{z}_{N}, \boldsymbol{w}_{N}\right)\right)_{N}=\left(\left(\boldsymbol{\vartheta}_{N}, A_{N}\left(\boldsymbol{w}_{N}\right)\right)\right)_{N}
$$

Since the norms $\|\cdot\|_{H(\operatorname{div}, \Omega)}$ and $\|\cdot\|_{L^{2}(\Omega)^{3}}$ are equal on $V_{N}$, it follows from (3.7) that the bilinear form in the left-hand side is elliptic on $V_{N}$, so that this problem has a unique solution $\boldsymbol{z}_{N}$. Moreover, this function satisfies for any $\varphi_{N}$ in $\mathbb{C}_{N}$

$$
\left(\left(\boldsymbol{z}_{N}, \operatorname{curl} \boldsymbol{\varphi}_{N}\right)\right)_{N}=\left(\left(\boldsymbol{\vartheta}_{N}, A_{N}\left(\operatorname{curl} \boldsymbol{\varphi}_{N}\right)\right)\right)_{N}
$$

Note that $A_{N}\left(\operatorname{curl} \varphi_{N}\right)$ is equal to $\boldsymbol{\varphi}_{N}$ in dimension $d=2$, to the sum of $\boldsymbol{\varphi}_{N}$ and of the gradient of a function $\mu_{N}$ in $\mathbb{G}_{N}$ in dimension $d=3$. Then, it follows from the choice of $\boldsymbol{\vartheta}_{N}$, see (3.15), that

$$
\left(\left(\boldsymbol{z}_{N}, \operatorname{curl} \boldsymbol{\varphi}_{N}\right)\right)_{N}=\left(\left(\boldsymbol{\vartheta}_{N}, \boldsymbol{\varphi}_{N}\right)\right)_{N}
$$

So the pair $\left(\boldsymbol{\vartheta}_{N}, \boldsymbol{z}_{N}\right)$ belongs to $\mathcal{W}_{N}$ and taking $\left(\boldsymbol{\omega}_{N}, \boldsymbol{u}_{N}\right)$ equal to $\left(\boldsymbol{\vartheta}_{N}, \boldsymbol{z}_{N}\right)$ yields thanks to (3.7) that $\boldsymbol{v}_{N}=\operatorname{curl} \boldsymbol{\vartheta}_{N}$ is zero, which concludes the proof.

Lemma 3.5. There exists a positive constant $\alpha_{*}$ independent of $N$ such that the form $a_{N}(\cdot, \cdot ; \cdot)$ satisfies the inf-sup condition

$$
\begin{aligned}
\forall\left(\boldsymbol{\omega}_{N}, \boldsymbol{u}_{N}\right) \in \mathcal{W}_{N}, \\
\sup _{\boldsymbol{v}_{N} \in V_{N}} \frac{a_{N}\left(\boldsymbol{\omega}_{N}, \boldsymbol{u}_{N} ; \boldsymbol{v}_{N}\right)}{\left\|\boldsymbol{v}_{N}\right\|_{L^{2}(\Omega)^{d}}} \geq \alpha_{*}\left(\left\|\boldsymbol{\omega}_{N}\right\|_{H(\operatorname{curl}, \Omega)}+\left\|\boldsymbol{u}_{N}\right\|_{L^{2}(\Omega)^{d}}\right) .
\end{aligned}
$$

Proof. For any $\left(\boldsymbol{\omega}_{N}, \boldsymbol{u}_{N}\right)$ in $\mathcal{W}_{N}$, we set $\boldsymbol{v}_{N}=\boldsymbol{u}_{N}+\operatorname{curl} \boldsymbol{\omega}_{N}$ and observe that it belongs to $V_{N}$. Next, we have

$$
a_{N}\left(\boldsymbol{\omega}_{N}, \boldsymbol{u}_{N} ; \boldsymbol{v}_{N}\right)=\nu\left(\left(\operatorname{curl} \boldsymbol{\omega}_{N}, \boldsymbol{u}_{N}\right)\right)_{N}+\nu\left(\left(\operatorname{curl} \boldsymbol{\omega}_{N}, \operatorname{curl} \boldsymbol{\omega}_{N}\right)\right)_{N}
$$


Thanks to the definition of $\mathcal{W}_{N}$, we have

$$
\left(\left(\operatorname{curl} \boldsymbol{\omega}_{N}, \boldsymbol{u}_{N}\right)\right)_{N}=\left(\left(\boldsymbol{\omega}_{N}, \boldsymbol{\omega}_{N}\right)\right)_{N}
$$

Combining this with (3.7) leads to

$$
a_{N}\left(\boldsymbol{\omega}_{N}, \boldsymbol{u}_{N} ; \boldsymbol{v}_{N}\right) \geq \nu\left\|\boldsymbol{\omega}_{N}\right\|_{H(\operatorname{curl}, \Omega)}^{2} .
$$

On the other hand, using once more the definition of $\mathcal{W}_{N}$ and (3.7), we write

$$
\begin{aligned}
\left\|\boldsymbol{u}_{N}\right\|_{L^{2}(\Omega)^{d}}^{2} \leq\left(\left(\boldsymbol{u}_{N}, \operatorname{curl} A_{N}\left(\boldsymbol{u}_{N}\right)\right)\right)_{N}= & \left(\left(\boldsymbol{\omega}_{N}, A_{N}\left(\boldsymbol{u}_{N}\right)\right)\right)_{N} \\
& \leq 3^{d}\left\|\boldsymbol{\omega}_{N}\right\|_{L^{2}(\Omega)^{\frac{d(d-1)}{2}}}\left\|A_{N}\left(\boldsymbol{u}_{N}\right)\right\|_{L^{2}(\Omega)} \frac{d(d-1)}{2} .
\end{aligned}
$$

So, we derive from (3.16) that

$$
\left\|\boldsymbol{u}_{N}\right\|_{L^{2}(\Omega)^{d}} \leq 3^{d} c\left\|\boldsymbol{\omega}_{N}\right\|_{L^{2}(\Omega) \frac{d(d-1)}{2}},
$$

whence

$$
a_{N}\left(\boldsymbol{\omega}_{N}, \boldsymbol{u}_{N} ; \boldsymbol{v}_{N}\right) \geq \frac{\nu}{2}\left\|\boldsymbol{\omega}_{N}\right\|_{H(\operatorname{curl}, \Omega)}^{2}+c^{\prime} \nu\left\|\boldsymbol{u}_{N}\right\|_{L^{2}(\Omega)^{d}}^{2} .
$$

We also have

$$
\left\|\boldsymbol{v}_{N}\right\|_{L^{2}(\Omega)^{d}} \leq \sqrt{2}\left(\left\|\boldsymbol{\omega}_{N}\right\|_{H(\mathbf{c u r l}, \Omega)}^{2}+\left\|\boldsymbol{u}_{N}\right\|_{L^{2}(\Omega)^{d}}^{2}\right)^{\frac{1}{2}} .
$$

Combining the last two inequalities gives the desired inf-sup condition.

The following result is a direct consequence of Lemmas 3.4 and 3.5, see [15], Chapter I, Lemma 4.1. Let $\mathcal{I}_{N}^{k}$ denote the Lagrange interpolation operator at the nodes $F_{k}\left(\xi_{i}, \xi_{j}\right)$ in dimension $d=2$ and $F_{k}\left(\xi_{i}, \xi_{j}, \xi_{p}\right)$ in dimension $d=3$, with values in $\mathbb{P}_{N}\left(\Omega_{k}\right)$ and $\mathcal{I}_{N}$ the global interpolation operator, defined on continuous functions $f$ by $\left.\left(\mathcal{I}_{N} f\right)\right|_{\Omega_{k}}=\left.\mathcal{I}_{N}^{k} f\right|_{\Omega_{k}}, 1 \leq k \leq K$. The following property is then easily derived from (3.7): For any $\boldsymbol{v}_{N}$ in $\mathbb{D}_{N}$,

$$
\left(\left(\boldsymbol{f}, \boldsymbol{v}_{N}\right)\right)_{N}=\left(\left(\mathcal{I}_{N} \boldsymbol{f}, \boldsymbol{v}_{N}\right)\right)_{N} \leq 3^{d}\left\|\mathcal{I}_{N} \boldsymbol{f}\right\|_{L^{2}(\Omega)^{d}}\left\|\boldsymbol{v}_{N}\right\|_{L^{2}(\Omega)^{d}} .
$$

Corollary 3.6. For any data $\boldsymbol{f}$ continuous on $\bar{\Omega}$, problem $(3.24)$ has a unique solution $\left(\boldsymbol{\omega}_{N}, \boldsymbol{u}_{N}\right)$ in $\mathcal{W}_{N}$. Moreover this solution satisfies for a constant $c$ independent of $N$

$$
\left\|\boldsymbol{\omega}_{N}\right\|_{H(\mathbf{c u r l}, \Omega)}+\left\|\boldsymbol{u}_{N}\right\|_{L^{2}(\Omega)^{d}} \leq c\left\|\mathcal{I}_{N} \boldsymbol{f}\right\|_{L^{2}(\Omega)^{d}} .
$$

In order to go further, we now establish an inf-sup condition on the form $b_{N}(\cdot, \cdot)$. It relies on the Boland and Nicolaides argument [8] and requires a standard finite element result, which involves the Nédélec operator [16], Section 2, but is much simpler here since the constant can depend on the size of the $\Omega_{k}$ (nevertheless, it requires that the $\Sigma_{j}$ are the union of faces of the subdomains). We refer to [17] for the first proof of this result.

Lemma 3.7. There exists a positive constant $\beta_{\sharp}$ such that the form $b(\cdot, \cdot ; \cdot \cdot)$ satisfies the inf-sup condition

$$
\forall q \in \mathbb{M}_{1}, \quad \sup _{\boldsymbol{v} \in \mathbb{D}_{1}} \frac{b(\boldsymbol{v}, q)}{\|\boldsymbol{v}\|_{H(\operatorname{div}, \Omega)}} \geq \beta_{\sharp}\|q\|_{L^{2}(\Omega)} .
$$

Lemma 3.8. There exists a positive constant $\beta_{*}$ independent of $N$ such that the form $b_{N}(\cdot, \cdot ; \cdot)$ satisfies the inf-sup condition

$$
\forall q_{N} \in \mathbb{M}_{N}, \quad \sup _{\boldsymbol{v}_{N} \in \mathbb{D}_{N}} \frac{b_{N}\left(\boldsymbol{v}_{N}, q_{N}\right)}{\left\|\boldsymbol{v}_{N}\right\|_{H(\operatorname{div}, \Omega)}} \geq \beta_{*}\left\|q_{N}\right\|_{L^{2}(\Omega)} .
$$


Proof. We recall that the forms $b(\cdot, \cdot)$ and $b_{N}(\cdot, \cdot)$ coincide on $\mathbb{D}_{N} \times \mathbb{M}_{N}$, so that we work with the form $b(\cdot, \cdot)$. Any $q_{N}$ in $\mathbb{M}_{N}$ admits the expansion

$$
q_{N}=\tilde{q}_{N}+\bar{q}_{N}, \quad \text { with }\left.\quad \bar{q}_{N}\right|_{\Omega_{k}}=\frac{1}{\operatorname{meas}\left(\Omega_{k}\right)} \int_{\Omega_{k}} q_{N}(\boldsymbol{x}) \mathrm{d} \boldsymbol{x}, \quad 1 \leq k \leq K .
$$

Then, each $\left.\tilde{q}_{N}\right|_{\Omega_{k}}$ belongs to $M_{N}^{k} \cap L_{0}^{2}\left(\Omega_{k}\right)$. So, using an appropriate mapping that sends the reference domain ] $-1,1\left[{ }^{d}\right.$ onto $\Omega_{k}$, it follows from [4], Lemma 3.9, that there exists a function $\boldsymbol{v}_{N}^{k}$ in $D_{N}^{k} \cap H_{0}\left(\operatorname{div}, \Omega_{k}\right)$ such that

$$
\operatorname{div} \boldsymbol{v}_{N}^{k}=-\left.\tilde{q}_{N}\right|_{\Omega_{k}} \quad \text { and } \quad\left\|\boldsymbol{v}_{N}^{k}\right\|_{H\left(\operatorname{div}, \Omega_{k}\right)} \leq \beta_{k}^{-1}\left\|\tilde{q}_{N}\right\|_{L^{2}\left(\Omega_{k}\right)},
$$

for a constant $\beta_{k}$ only depending on $\Omega_{k}$. We thus define the function $\tilde{\boldsymbol{v}}_{N}$ such that each $\left.\tilde{\boldsymbol{v}}_{N}\right|_{\Omega_{k}}$ is equal to $\boldsymbol{v}_{N}^{k}$, $1 \leq k \leq K$, and observe that, since the $\Sigma_{j}$ are the union of faces of some $\Omega_{k}, \tilde{\boldsymbol{v}}_{N} \cdot \boldsymbol{n}$ vanishes on $\Sigma_{j}$, so that $\tilde{\boldsymbol{v}}_{N}$ belongs to $\mathbb{D}_{N}$. On the other hand, since $\bar{q}_{N}$ belongs to $L_{0}^{2}(\Omega)$ and is constant on each $\Omega_{k}$, hence to belongs to $\mathbb{M}_{1}$, Lemma 3.7 yields the existence of a function $\overline{\boldsymbol{v}}$ in $\mathbb{D}_{1}$ such that

$$
\operatorname{div} \overline{\boldsymbol{v}}=-\left.\bar{q}_{N}\right|_{\Omega_{k}} \quad \text { and } \quad\|\overline{\boldsymbol{v}}\|_{H(\operatorname{div}, \Omega)} \leq \beta_{\sharp}^{-1}\left\|\bar{q}_{N}\right\|_{L^{2}(\Omega)} .
$$

The argument of Boland and Nicolaides consists now in taking $\boldsymbol{v}_{N}=\tilde{\boldsymbol{v}}_{N}+\lambda \overline{\boldsymbol{v}}$, for a positive constant $\lambda$. Indeed, it can be checked by integration by parts on each $\Omega_{k}$ that $b\left(\tilde{\boldsymbol{v}}_{N}, \bar{q}_{N}\right)$ is equal to zero, so that, thanks to the choice of $\tilde{\boldsymbol{v}}_{N}$ and $\overline{\boldsymbol{v}}$,

$$
b\left(\boldsymbol{v}_{N}, q_{N}\right) \geq\left\|\tilde{q}_{N}\right\|_{L^{2}(\Omega)}^{2}+\lambda\left\|\bar{q}_{N}\right\|_{L^{2}(\Omega)}^{2}-\lambda\|\overline{\boldsymbol{v}}\|_{H(\operatorname{div}, \Omega)}\left\|\tilde{q}_{N}\right\|_{L^{2}(\Omega)} .
$$

This yields

$$
\begin{aligned}
b\left(\boldsymbol{v}_{N}, q_{N}\right) \geq\left\|\tilde{q}_{N}\right\|_{L^{2}(\Omega)}^{2}+\lambda\left\|\bar{q}_{N}\right\|_{L^{2}(\Omega)}^{2}-\lambda \beta_{\sharp}^{-1}\left\|\bar{q}_{N}\right\|_{L^{2}(\Omega)}\left\|\tilde{q}_{N}\right\|_{L^{2}(\Omega)} \\
\geq \frac{1}{2}\left\|\tilde{q}_{N}\right\|_{L^{2}(\Omega)}^{2}+\lambda\left(1-\frac{\lambda}{2 \beta_{\sharp}^{2}}\right)\left\|\bar{q}_{N}\right\|_{L^{2}(\Omega)}^{2} .
\end{aligned}
$$

We now take $\lambda$ equal to $\beta_{\sharp}^{2}$, so that

$$
b\left(\boldsymbol{v}_{N}, q_{N}\right) \geq \frac{1}{2} \min \left\{1, \beta_{\sharp}^{2}\right\}\left(\left\|\tilde{q}_{N}\right\|_{L^{2}(\Omega)}^{2}+\left\|\bar{q}_{N}\right\|_{L^{2}(\Omega)}^{2}\right) .
$$

We also have

$$
\left\|\boldsymbol{v}_{N}\right\|_{H(\operatorname{div}, \Omega)} \leq\left(\max _{1 \leq k \leq K} \beta_{k}^{-1}\right)\left\|\tilde{q}_{N}\right\|_{L^{2}(\Omega)}+\beta_{\sharp}\left\|\bar{q}_{N}\right\|_{L^{2}(\Omega)} .
$$

The two previous inequalities, when combined with the orthogonality property

$$
\left\|q_{N}\right\|_{L^{2}(\Omega)}^{2}=\left\|\tilde{q}_{N}\right\|_{L^{2}(\Omega)}^{2}+\left\|\bar{q}_{N}\right\|_{L^{2}(\Omega)}^{2},
$$

lead to the desired inf-sup condition.

The proof of the final theorem is now completely standard, see [15], Chapter I, Lemma 4.1, for instance.

Theorem 3.9. For any data $\boldsymbol{f}$ continuous on $\bar{\Omega}$, problem (3.10) has a unique solution $\left(\boldsymbol{\omega}_{N}, \boldsymbol{u}_{N}, p_{N}\right)$ in $\mathbb{C}_{N} \times$ $\mathbb{D}_{N} \times \mathbb{M}_{N}$. Moreover this solution satisfies for a constant $c$ independent of $N$

$$
\left\|\boldsymbol{\omega}_{N}\right\|_{H(\mathbf{c u r l}, \Omega)}+\left\|\boldsymbol{u}_{N}\right\|_{H(\mathrm{div}, \Omega)}+\left\|p_{N}\right\|_{L^{2}(\Omega)} \leq c\left\|\mathcal{I}_{N} \boldsymbol{f}\right\|_{L^{2}(\Omega)^{d}} .
$$




\section{ERror estimates}

We now wish to derive the error estimates between the solution $(\boldsymbol{\omega}, \boldsymbol{u}, p)$ of problem (2.9) and the solution $\left(\boldsymbol{\omega}_{N}, \boldsymbol{u}_{N}, p_{N}\right)$ of problem (3.10). The arguments are very similar to their analogues in the case of one element, see [4], Section 4, and require several lemmas. In all that follows, $c$ stands for a generic constant which can vary from one line to the next one but is always independent of $N$.

Lemma 4.1. The following estimate holds for the error between the solution $(\boldsymbol{\omega}, \boldsymbol{u}, p)$ of problem (2.9) and the solution $\left(\boldsymbol{\omega}_{N}, \boldsymbol{u}_{N}, p_{N}\right)$ of problem $(3.10)$ :

$$
\begin{aligned}
\left\|\boldsymbol{\omega}-\boldsymbol{\omega}_{N}\right\|_{H(\operatorname{curl}, \Omega)}+\left\|\boldsymbol{u}-\boldsymbol{u}_{N}\right\|_{H(\operatorname{div}, \Omega)} & \\
\leq c \inf _{\left(\boldsymbol{\vartheta}_{N}, \boldsymbol{w}_{N}\right) \in \mathcal{W}_{N}}\left(\left\|\boldsymbol{\omega}-\boldsymbol{\vartheta}_{N}\right\|_{H(\operatorname{curl}, \Omega)}+\left\|\boldsymbol{u}-\boldsymbol{w}_{N}\right\|_{L^{2}(\Omega)^{d}}\right. & \left.+E_{N}^{\boldsymbol{f}}+E_{N}^{a}\left(\boldsymbol{\vartheta}_{N}, \boldsymbol{w}_{N}\right)\right),
\end{aligned}
$$

where the quantities $E_{N}^{f}$ and $E_{N}^{a}\left(\boldsymbol{\vartheta}_{N}, \boldsymbol{w}_{N}\right)$ are defined by

$$
\begin{aligned}
E_{N}^{\boldsymbol{f}}=\sup _{\boldsymbol{v}_{N} \in \mathbb{D}_{N}} \frac{\left\langle\boldsymbol{f}, \boldsymbol{v}_{N}\right\rangle-\left(\left(\boldsymbol{f}, \boldsymbol{v}_{N}\right)\right)_{N}}{\left\|\boldsymbol{v}_{N}\right\|_{L^{2}(\Omega)^{d}}} & \\
E_{N}^{a}\left(\boldsymbol{\vartheta}_{N}, \boldsymbol{w}_{N}\right) & =\sup _{\boldsymbol{v}_{N} \in \mathbb{D}_{N}} \frac{\left(a-a_{N}\right)\left(\boldsymbol{\vartheta}_{N}, \boldsymbol{w}_{N} ; \boldsymbol{v}_{N}\right)}{\left\|\boldsymbol{v}_{N}\right\|_{L^{2}(\Omega)^{d}}} .
\end{aligned}
$$

Proof. Let $\left(\boldsymbol{\vartheta}_{N}, \boldsymbol{w}_{N}\right)$ be an approximation of $(\boldsymbol{\omega}, \boldsymbol{u})$ in $\mathcal{W}_{N}$. It follows from (3.24) that, for all $\boldsymbol{v}_{N}$ in $V_{N}$,

$$
a_{N}\left(\boldsymbol{\omega}_{N}-\boldsymbol{\vartheta}_{N}, \boldsymbol{u}_{N}-\boldsymbol{w}_{N} ; \boldsymbol{v}_{N}\right)=\left(\left(\boldsymbol{f}, \boldsymbol{v}_{N}\right)\right)_{N}-a_{N}\left(\boldsymbol{\vartheta}_{N}, \boldsymbol{w}_{N} ; \boldsymbol{v}_{N}\right) .
$$

Then, using problem (2.9) (we recall that $V_{N}$ is contained in $V$ ) leads to

$$
\begin{array}{r}
a_{N}\left(\boldsymbol{\omega}_{N}-\boldsymbol{\vartheta}_{N}, \boldsymbol{u}_{N}-\boldsymbol{w}_{N} ; \boldsymbol{v}_{N}\right)=\left(\left(\boldsymbol{f}, \boldsymbol{v}_{N}\right)\right)_{N}-\left\langle\boldsymbol{f}, \boldsymbol{v}_{N}\right\rangle+a\left(\boldsymbol{\omega}-\boldsymbol{\vartheta}_{N}, \boldsymbol{u}-\boldsymbol{w}_{N} ; \boldsymbol{v}_{N}\right) \\
+\left(a-a_{N}\right)\left(\boldsymbol{\vartheta}_{N}, \boldsymbol{w}_{N} ; \boldsymbol{v}_{N}\right) .
\end{array}
$$

When using the inf-sup condition (3.27), we derive

$$
\left\|\boldsymbol{\omega}_{N}-\boldsymbol{\vartheta}_{N}\right\|_{H(\operatorname{curl}, \Omega)}+\left\|\boldsymbol{u}_{N}-\boldsymbol{w}_{N}\right\|_{L^{2}(\Omega)^{d}} \leq c\left(\left\|\operatorname{curl}\left(\boldsymbol{\omega}-\boldsymbol{\vartheta}_{N}\right)\right\|_{L^{2}(\Omega)^{d}}+E_{N}^{\boldsymbol{f}}+E_{N}^{a}\left(\boldsymbol{\vartheta}_{N}, \boldsymbol{w}_{N}\right)\right) .
$$

We conclude thanks to a triangle inequality, by noting that both $\boldsymbol{u}$ and $\boldsymbol{u}_{N}$ are exactly divergence-free.

Lemma 4.2. The following estimate holds for the error between the solution $(\boldsymbol{\omega}, \boldsymbol{u}, p)$ of problem (2.9) and the solution $\left(\boldsymbol{\omega}_{N}, \boldsymbol{u}_{N}, p_{N}\right)$ of problem $(3.10)$ :

$$
\begin{aligned}
\left\|p-p_{N}\right\|_{L^{2}(\Omega)} \leq c \inf _{q_{N} \in \mathbb{M}_{N}}\left\|p-q_{N}\right\|_{L^{2}(\Omega)} & \\
+c \inf _{\left(\boldsymbol{\vartheta}_{N}, \boldsymbol{w}_{N}\right) \in \mathcal{W}_{N}}\left(\left\|\boldsymbol{\omega}-\boldsymbol{\vartheta}_{N}\right\|_{H(\operatorname{curl}, \Omega)}+\left\|\boldsymbol{u}-\boldsymbol{w}_{N}\right\|_{L^{2}(\Omega)^{d}}\right. & \left.+E_{N}^{\boldsymbol{f}}+E_{N}^{a}\left(\boldsymbol{\vartheta}_{N}, \boldsymbol{w}_{N}\right)\right),
\end{aligned}
$$

where the quantities $E_{N}^{\boldsymbol{f}}$ and $E_{N}^{a}\left(\boldsymbol{\vartheta}_{N}, \boldsymbol{w}_{N}\right)$ are defined in (4.2). 
Proof. It follows from problems $(2.9)$ and $(3.10)$ (note also that $b(\cdot, \cdot)$ and $b_{N}(\cdot, \cdot)$ coincide on $\left.\mathbb{D}_{N} \times \mathbb{M}_{N}\right)$ that, for any $\boldsymbol{v}_{N}$ in $\mathbb{D}_{N}$ and $q_{N}$ in $\mathbb{M}_{N}$,

$$
\begin{aligned}
b_{N}\left(\boldsymbol{v}_{N}, p_{N}-q_{N}\right)=\left(\left(\boldsymbol{f}, \boldsymbol{v}_{N}\right)\right)_{N}-\left\langle\boldsymbol{f}, \boldsymbol{v}_{N}\right\rangle & +a\left(\boldsymbol{\omega}-\boldsymbol{\omega}_{N}, \boldsymbol{u}-\boldsymbol{u}_{N} ; \boldsymbol{v}_{N}\right) \\
& +\left(a-a_{N}\right)\left(\boldsymbol{\omega}_{N}, \boldsymbol{u}_{N} ; \boldsymbol{v}_{N}\right)+b\left(\boldsymbol{v}_{N}, p-q_{N}\right) .
\end{aligned}
$$

Moreover, we use the identity

$$
\left(a-a_{N}\right)\left(\boldsymbol{\omega}_{N}, \boldsymbol{u}_{N} ; \boldsymbol{v}_{N}\right)=\left(a-a_{N}\right)\left(\boldsymbol{\vartheta}_{N}, \boldsymbol{w}_{N} ; \boldsymbol{v}_{N}\right)+\left(a-a_{N}\right)\left(\boldsymbol{\omega}_{N}-\boldsymbol{\vartheta}_{N}, \boldsymbol{u}_{N}-\boldsymbol{w}_{N} ; \boldsymbol{v}_{N}\right) .
$$

So, the inf-sup condition (3.30) combined with Lemma 4.1 and the fact that the norm of $a_{N}(\cdot, \cdot ; \cdot)$ is bounded independently of $N$ leads to the desired estimate.

In order to evaluate the distance of $(\boldsymbol{\omega}, \boldsymbol{u})$ to $\mathcal{W}_{N}$, we now prove an inf-sup condition on the form $c_{N}(\cdot, \cdot ; \cdot)$.

Lemma 4.3. There exists a positive constant $\gamma_{*}$ independent of $N$ such that the form $c_{N}(\cdot, \cdot ; \cdot)$ satisfies the inf-sup condition

$$
\forall \boldsymbol{\varphi}_{N} \in \mathbb{C}_{N}, \quad \sup _{\left(\boldsymbol{\omega}_{N}, \boldsymbol{u}_{N}\right) \in \mathbb{C}_{N} \times V_{N}} \frac{c_{N}\left(\boldsymbol{\omega}_{N}, \boldsymbol{u}_{N} ; \boldsymbol{\varphi}_{N}\right)}{\left\|\boldsymbol{\omega}_{N}\right\|_{H(\operatorname{curl}, \Omega)}+\left\|\boldsymbol{u}_{N}\right\|_{L^{2}(\Omega)^{d}}} \geq \gamma_{*}\left\|\boldsymbol{\varphi}_{N}\right\|_{H(\operatorname{curl}, \Omega)} .
$$

Proof. For any $\boldsymbol{\varphi}_{N}$ in $\mathbb{C}_{N}$, we take $\left(\boldsymbol{\omega}_{N}, \boldsymbol{u}_{N}\right)$ equal to $\left(\boldsymbol{\varphi}_{N},-\operatorname{curl} \boldsymbol{\varphi}_{N}\right)$ and recall that it belongs to $\mathbb{C}_{N} \times V_{N}$ (see for instance [2], form. (3.15)). Next, we derive from (3.7) that

$$
c_{N}\left(\boldsymbol{\omega}_{N}, \boldsymbol{u}_{N} ; \boldsymbol{\varphi}_{N}\right)=\left(\left(\boldsymbol{\varphi}_{N}, \boldsymbol{\varphi}_{N}\right)\right)_{N}+\left(\left(\operatorname{curl} \boldsymbol{\varphi}_{N}, \operatorname{curl} \boldsymbol{\varphi}_{N}\right)\right)_{N} \geq\left\|\boldsymbol{\varphi}_{N}\right\|_{H(\operatorname{curl}, \Omega)}^{2} .
$$

On the other hand, we have

$$
\left\|\boldsymbol{\omega}_{N}\right\|_{H(\operatorname{curl}, \Omega)}+\left\|\boldsymbol{u}_{N}\right\|_{L^{2}(\Omega) \mathrm{d}} \leq 2\left\|\boldsymbol{\varphi}_{N}\right\|_{H(\operatorname{curl}, \Omega)},
$$

which leads to the desired inf-sup condition.

Corollary 4.4. The following estimate holds

$$
\begin{aligned}
& \quad \inf _{\left(\boldsymbol{\vartheta}_{N}, \boldsymbol{w}_{N}\right) \in \mathcal{W}_{N}}\left(\left\|\boldsymbol{\omega}-\boldsymbol{\vartheta}_{N}\right\|_{H(\operatorname{curl}, \Omega)}+\left\|\boldsymbol{u}-\boldsymbol{w}_{N}\right\|_{L^{2}(\Omega)^{d}}\right) \\
& \quad \leq c \inf _{\left(\boldsymbol{\zeta}_{N}, \boldsymbol{z}_{N}\right) \in \mathbb{C}_{N} \times V_{N}}\left(\left\|\boldsymbol{\omega}-\boldsymbol{\zeta}_{N}\right\|_{H(\operatorname{curl}, \Omega)}+\left\|\boldsymbol{u}-\boldsymbol{z}_{N}\right\|_{L^{2}(\Omega)^{d}}+E_{N}^{c}\left(\boldsymbol{\zeta}_{N}, \boldsymbol{z}_{N}\right)\right),
\end{aligned}
$$

where the quantity $E_{N}^{c}\left(\boldsymbol{\zeta}_{N}, \boldsymbol{z}_{N}\right)$ is defined by

$$
E_{N}^{c}\left(\boldsymbol{\zeta}_{N}, \boldsymbol{z}_{N}\right)=\sup _{\boldsymbol{\varphi}_{N} \in \mathbb{C}_{N}} \frac{\left(c-c_{N}\right)\left(\boldsymbol{\zeta}_{N}, \boldsymbol{z}_{N} ; \boldsymbol{\varphi}_{N}\right)}{\left\|\boldsymbol{\varphi}_{N}\right\|_{H(\operatorname{curl}, \Omega)}}
$$

Proof. For any $\left(\boldsymbol{\zeta}_{N}, \boldsymbol{z}_{N}\right)$ on $\mathbb{C}_{N} \times V_{N}$, we derive from the inf-sup condition (4.4) the existence of a pair $\left(\tilde{\boldsymbol{\zeta}}_{N}, \tilde{\boldsymbol{z}}_{N}\right)$ also in $\mathbb{C}_{N} \times V_{N}$ which satisfies for all $\varphi_{N}$ in $\mathbb{C}_{N}$

$$
c_{N}\left(\tilde{\boldsymbol{\zeta}}_{N}, \tilde{\boldsymbol{z}}_{N} ; \boldsymbol{\varphi}_{N}\right)=c_{N}\left(\boldsymbol{\zeta}_{N}, \boldsymbol{z}_{N} ; \boldsymbol{\varphi}_{N}\right),
$$

and moreover

$$
\left\|\tilde{\boldsymbol{\zeta}}_{N}\right\|_{H(\operatorname{curl}, \Omega)}+\left\|\tilde{\boldsymbol{z}}_{N}\right\|_{L^{2}(\Omega)^{d}} \leq \gamma_{*}^{-1} \sup _{\boldsymbol{\varphi}_{N} \in \mathbb{C}_{N}} \frac{c_{N}\left(\boldsymbol{\zeta}_{N}, \boldsymbol{z}_{N} ; \boldsymbol{\varphi}_{N}\right)}{\left\|\boldsymbol{\varphi}_{N}\right\|_{H(\operatorname{curl}, \Omega)}}
$$


We also note that

$$
c_{N}\left(\boldsymbol{\zeta}_{N}, \boldsymbol{z}_{N} ; \boldsymbol{\varphi}_{N}\right)=-c\left(\boldsymbol{\omega}-\boldsymbol{\zeta}_{N}, \boldsymbol{u}-\boldsymbol{z}_{N} ; \boldsymbol{\varphi}_{N}\right)-\left(c-c_{N}\right)\left(\boldsymbol{\zeta}_{N}, \boldsymbol{z}_{N} ; \boldsymbol{\varphi}_{N}\right) .
$$

Since the pair $\left(\boldsymbol{\vartheta}_{N}, \boldsymbol{w}_{N}\right)$ with $\boldsymbol{\vartheta}_{N}=\boldsymbol{\zeta}_{N}-\tilde{\boldsymbol{\zeta}}_{N}$ and $\boldsymbol{w}_{N}=\boldsymbol{z}_{N}-\tilde{\boldsymbol{z}}_{N}$ belongs to $\mathcal{W}_{N}$, the desired estimate is easily derived from the two previous lines.

By combining Lemmas 4.1 and 4.2 and Corollary 4.4, we observe that the full error

$$
\left\|\boldsymbol{\omega}-\boldsymbol{\omega}_{N}\right\|_{H(\operatorname{curl}, \Omega)}+\left\|\boldsymbol{u}-\boldsymbol{u}_{N}\right\|_{H(\operatorname{div}, \Omega)}+\left\|p-p_{N}\right\|_{L^{2}(\Omega)}
$$

is bounded by the sum of the three terms of approximation error

$$
\inf _{\boldsymbol{\zeta}_{N} \in \mathbb{C}_{N}}\left\|\boldsymbol{\omega}-\boldsymbol{\zeta}_{N}\right\|_{H(\operatorname{curl}, \Omega)}, \quad \inf _{\boldsymbol{z}_{N} \in V_{N}}\left\|\boldsymbol{u}-\boldsymbol{z}_{N}\right\|_{L^{2}(\Omega)^{d}}, \quad \inf _{q_{N} \in \mathbb{M}_{N}}\left\|p-q_{N}\right\|_{L^{2}(\Omega)},
$$

plus the three quantities $E_{N}^{\boldsymbol{f}}, E_{N}^{a}\left(\boldsymbol{\vartheta}_{N}, \boldsymbol{w}_{N}\right)$ and $E_{N}^{c}\left(\boldsymbol{\zeta}_{N}, \boldsymbol{z}_{N}\right)$ which are issued from numerical integration.

In order to estimate these last ones, we introduce the orthogonal projection operator $\Pi_{N-1}^{k}$ from $L^{2}\left(\Omega_{k}\right)$ onto $\mathbb{P}_{N-1}\left(\Omega_{k}\right)$ and we denote by $\mathcal{I}_{N}^{k}$ the Lagrange interpolation operator at the nodes $F_{k}\left(\xi_{i}, \xi_{j}\right)$ in dimension $d=2$, at the nodes $F_{k}\left(\xi_{i}, \xi_{j}, \xi_{p}\right)$ in dimension $d=3$, with values in $\mathbb{P}_{N}\left(\Omega_{k}\right)$. Indeed, using (3.6) leads to, for any $\boldsymbol{v}_{N}$ in $\mathbb{D}_{N}$,

$$
\begin{aligned}
& \int_{\Omega_{k}} \boldsymbol{f}(\boldsymbol{x}) \cdot \boldsymbol{v}_{N}(\boldsymbol{x}) \mathrm{d} \boldsymbol{x}-\left(\boldsymbol{f}, \boldsymbol{v}_{N}\right)_{N}^{k} \\
& \quad=\int_{\Omega_{k}}\left(\boldsymbol{f}-\Pi_{N-1}^{k} \boldsymbol{f}\right)(\boldsymbol{x}) \cdot \boldsymbol{v}_{N}(\boldsymbol{x}) \mathrm{d} \boldsymbol{x}-\left(\mathcal{I}_{N}^{k} \boldsymbol{f}-\Pi_{N-1}^{k} \boldsymbol{f}, \boldsymbol{v}_{N}\right)_{N}^{k},
\end{aligned}
$$

so that, owing to (3.7),

$$
E_{N}^{\boldsymbol{f}} \leq c \sum_{k=1}^{K}\left(\left\|\boldsymbol{f}-\Pi_{N-1}^{k} \boldsymbol{f}\right\|_{L^{2}\left(\Omega_{k}\right)^{d}}+\left\|\boldsymbol{f}-\mathcal{I}_{N}^{k} \boldsymbol{f}\right\|_{L^{2}\left(\Omega_{k}\right)^{d}}\right) .
$$

Similarly, we have for any $\boldsymbol{v}_{N}$ in $\mathbb{D}_{N}$

$$
\begin{aligned}
\left(a-a_{N}\right)\left(\boldsymbol{\vartheta}_{N}, \boldsymbol{z}_{N} ; \boldsymbol{v}_{N}\right)=\nu \sum_{k=1}^{N}\left(\int_{\Omega}\left(\operatorname{curl} \boldsymbol{\vartheta}_{N}-\Pi_{N-1}^{k}(\operatorname{curl} \boldsymbol{\omega})\right)(\boldsymbol{x}) \cdot \boldsymbol{z}_{N}(\boldsymbol{x}) \mathrm{d} \boldsymbol{x}\right. \\
\left.-\nu\left(\operatorname{curl} \boldsymbol{\vartheta}_{N}-\Pi_{N-1}^{k}(\operatorname{curl} \boldsymbol{\omega}), \boldsymbol{z}_{N}\right)_{N}^{k}\right),
\end{aligned}
$$

so that

$$
E_{N}^{a}\left(\boldsymbol{\vartheta}_{N}, \boldsymbol{w}_{N}\right) \leq c\left(\left\|\operatorname{curl}\left(\boldsymbol{\omega}-\boldsymbol{\vartheta}_{N}\right)\right\|_{L^{2}(\Omega)^{d}}+\sum_{k=1}^{K}\left\|\operatorname{curl} \boldsymbol{\omega}-\Pi_{N-1}^{k}(\operatorname{curl} \boldsymbol{\omega})\right\|_{L^{2}\left(\Omega_{k}\right)^{d}}\right) .
$$

Similar arguments also lead to

$$
\begin{array}{r}
E_{N}^{c}\left(\boldsymbol{\zeta}_{N}, \boldsymbol{z}_{N}\right) \leq c\left(\left\|\boldsymbol{\omega}-\boldsymbol{\zeta}_{N}\right\|_{L^{2}(\Omega)} \frac{d(d-1)}{2}+\sum_{k=1}^{K}\left\|\boldsymbol{\omega}-\Pi_{N-1}^{k} \boldsymbol{\omega}\right\|_{L^{2}\left(\Omega_{k} \frac{d(d-1)}{2}\right.}\right. \\
\left.+\left\|\boldsymbol{u}-\boldsymbol{z}_{N}\right\|_{L^{2}(\Omega)^{d}}+\sum_{k=1}^{K}\left\|\boldsymbol{u}-\Pi_{N-1}^{k} \boldsymbol{u}\right\|_{L^{2}\left(\Omega_{k}\right)^{d}}\right) .
\end{array}
$$


We recall from [5], Theorems 7.1 and 14.2, the approximation properties of the operators $\Pi_{N-1}^{k}$ and $\mathcal{I}_{N}^{k}$ : For any function $g$ in $H^{s}\left(\Omega_{k}\right), s \geq 0$,

$$
\left\|g-\Pi_{N-1}^{k} g\right\|_{L^{2}\left(\Omega_{k}\right)} \leq c N^{-s}\|g\|_{H^{s}\left(\Omega_{k}\right)},
$$

and, for any function $g$ in $H^{s}\left(\Omega_{k}\right), s>\frac{d}{2}$,

$$
\left\|g-\mathcal{I}_{N}^{k} g\right\|_{L^{2}\left(\Omega_{k}\right)} \leq c N^{-s}\|g\|_{H^{s}\left(\Omega_{k}\right)} .
$$

These estimates make complete the evaluation of $E_{N}^{f}$ and reduce the evaluation of both quantities $E_{N}^{a}\left(\boldsymbol{\vartheta}_{N}, \boldsymbol{w}_{N}\right)$ and $E_{N}^{c}\left(\boldsymbol{\zeta}_{N}, \boldsymbol{z}_{N}\right)$ to a bound for the approximation errors.

The approximation error for the pressure can also be estimated from (4.10). Indeed, since each $\Pi_{N-1}^{k}$ preserves the integral on $\Omega_{k}$, for each function $p$ in $L_{0}^{2}(\Omega)$, the function equal to $\Pi_{N-1}^{k} p$ on each $\Omega_{k}$ belongs to $\mathbb{M}_{N}$.

Lemma 4.5. For any function $p$ in $L_{0}^{2}(\Omega)$ such that each $\left.p\right|_{\Omega_{k}}, 1 \leq k \leq K$, belongs to $H^{s}\left(\Omega_{k}\right), s \geq 0$, the following estimate holds

$$
\inf _{q_{N} \in \mathbb{M}_{N}}\left\|p-q_{N}\right\|_{L^{2}(\Omega)} \leq c N^{-s} \sum_{k=1}^{K}\|p\|_{H^{s}\left(\Omega_{k}\right)} .
$$

Estimating the other approximation error terms requires some further local properties that we now state.

- In dimension $d=2$, the interpolation operator $\mathcal{I}_{N}^{k}$ satisfies [5], Theorem 14.2, for any function $g$ in $H^{s}\left(\Omega_{k}\right), s>\frac{3}{2}$,

$$
\left\|g-\mathcal{I}_{N}^{k} g\right\|_{H^{1}\left(\Omega_{k}\right)} \leq c N^{1-s}\|g\|_{H^{s}\left(\Omega_{k}\right)} .
$$

- In dimension $d=3$, a spectral analogue $\mathcal{R}_{N}^{k}$ of the Nédélec operator [16], Section 2, has been constructed in [3], Section 4. It maps smooth functions in $H\left(\operatorname{curl}, \Omega_{k}\right)$ onto the space $C_{N}^{k}$ defined in (3.2) and satisfies, for all functions $\varphi$ in $H^{s}\left(\Omega_{k}\right)^{3}, s \geq 2$,

$$
\left\|\boldsymbol{\varphi}-\mathcal{R}_{N}^{k} \boldsymbol{\varphi}\right\|_{L^{2}\left(\Omega_{k}\right)^{3}} \leq c N^{-s}\|\boldsymbol{\varphi}\|_{H^{s}\left(\Omega_{k}\right)^{3}},
$$

and, for all functions $\varphi$ in $H\left(\operatorname{curl}, \Omega_{k}\right)$ such that $\operatorname{curl} \varphi$ belongs to $H^{s}\left(\Omega_{k}\right)^{3}, s \geq \frac{3}{2}$,

$$
\left\|\operatorname{curl}\left(\boldsymbol{\varphi}-\mathcal{R}_{N}^{k} \boldsymbol{\varphi}\right)\right\|_{L^{2}\left(\Omega_{k}\right)^{3}} \leq c N^{-s}\|\operatorname{curl} \varphi\|_{H^{s}\left(\Omega_{k}\right)^{3}} .
$$

Moreover these operators satisfy the following properties on the boundary of $\partial \Omega_{k}$ : The trace of $\mathcal{I}_{N}^{k} g$ on each edge of $\Omega_{k}$ in dimension $d=2$ only depends on the trace of $g$ on this edge, the tangential trace of $\mathcal{R}_{N}^{k} \varphi$ on each face of $\Omega_{k}$ in dimension $d=3$ only depends on the tangential trace of $\boldsymbol{\varphi}$ on this face.

This leads to the next result in an obvious way. Indeed, for any function $\boldsymbol{\omega}$ in $H_{0}(\operatorname{curl}, \Omega)$, taking $\boldsymbol{\zeta}_{N}$ such that each $\left.\boldsymbol{\zeta}_{N}\right|_{\Omega_{k}}$ is equal to $\mathcal{I}_{N}^{k} \boldsymbol{\omega}$ in dimension $d=2, \mathcal{R}_{N}^{k} \boldsymbol{\omega}$ in dimension $d=3$ and using the previous properties yield that $\boldsymbol{\zeta}_{N}$ belongs to $\mathbb{C}_{N}$. The following statement requires the space, for $s \geq 0$,

$$
H^{s}\left(\operatorname{curl}, \Omega_{k}\right)=\left\{\varphi \in H^{s}\left(\Omega_{k}\right)^{\frac{d(d-1)}{2}} ; \operatorname{curl} \varphi \in H^{s}\left(\Omega_{k}\right)^{d}\right\} .
$$

Note that this space coincides with $H^{s+1}\left(\Omega_{k}\right)$ in dimension $d=2$.

Lemma 4.6. For any function $\boldsymbol{\omega}$ in $H_{0}(\mathbf{c u r l}, \Omega)$ such that each $\left.\boldsymbol{\omega}\right|_{\Omega_{k}}, 1 \leq k \leq K$, belongs to $H^{s}\left(\mathbf{c u r l}, \Omega_{k}\right)$, $s>\frac{d+1}{2}$, the following estimate holds

$$
\inf _{\boldsymbol{\zeta}_{N} \in \mathbb{C}_{N}}\left\|\boldsymbol{\omega}-\boldsymbol{\zeta}_{N}\right\|_{H(\operatorname{curl}, \Omega)} \leq c N^{-s} \sum_{k=1}^{K}\|\boldsymbol{\omega}\|_{H^{s}\left(\operatorname{curl}, \Omega_{k}\right)} .
$$


Finally, we recall [2], Theorem 3.17, that any function $\boldsymbol{u}$ in $V$ is equal to $\operatorname{curl} \boldsymbol{\psi}$, for a function $\boldsymbol{\psi}$ in $H_{0}(\operatorname{curl}, \Omega)$. Moreover, only in dimension $d=2$, if $\left.\boldsymbol{u}\right|_{\Omega_{k}}$ belongs to $H^{s}\left(\Omega_{k}\right),\left.\boldsymbol{\psi}\right|_{\Omega_{k}}$ belongs to $H^{s+1}\left(\Omega_{k}\right)$. So, an approximation $\boldsymbol{\psi}_{N}$ of $\boldsymbol{\psi}$ in $\mathbb{C}_{N}$ can be defined as equal to $\mathcal{I}_{N}^{k} \boldsymbol{\psi}(d=2)$ or to $\mathcal{R}_{N}^{k} \boldsymbol{\psi}(d=3)$ on each $\Omega_{k}$. It can also be noted that,

- in dimension $d=2$, the quantity $\left\langle\operatorname{curl} \psi_{N} \cdot \boldsymbol{n}, 1\right\rangle_{\Sigma_{j}}$, is equal to the difference of values of $\boldsymbol{\psi}_{N}$, hence of $\boldsymbol{\psi}$, between two $\Gamma_{i}$, so it is zero;

- in dimension $d=3$, the integral of $\operatorname{curl} \mathcal{R}_{N}^{k} \psi$ on each face of $\Omega_{k}$ is equal to the integral of $\operatorname{curl} \psi$ (see [3], Sect. 4),

so that the nullity of the $\langle\operatorname{curl} \psi \cdot \boldsymbol{n}, 1\rangle_{\Sigma_{j}}$ is preserved by this approximation. So, $\operatorname{curl} \boldsymbol{\psi}_{N}$ belongs to $V_{N}$, and the next estimate follows from (4.13) and (4.15).

Lemma 4.7. For any function $\boldsymbol{u}$ in $V$ such that each $\left.\boldsymbol{u}\right|_{\Omega_{k}}, 1 \leq k \leq K$, belongs to $H^{s}\left(\Omega_{k}\right), s>d-\frac{3}{2}$, the following estimate holds

$$
\inf _{\boldsymbol{z}_{N} \in V_{N}}\left\|\boldsymbol{u}-\boldsymbol{z}_{N}\right\|_{L^{2}(\Omega)^{d}} \leq c N^{-s} \sum_{k=1}^{K}\|\boldsymbol{u}\|_{H^{s}\left(\mathbf{c u r l}, \Omega_{k}\right)} .
$$

Theorem 4.8. Assume that the data $\boldsymbol{f}$ belong to $H^{\sigma}(\Omega)^{d}$ for a real number $\sigma>\frac{d}{2}$ and also that the solution $(\boldsymbol{\omega}, \boldsymbol{u}, p)$ of problem $(2.9)$ belongs to $H^{s}(\mathbf{c u r l}, \Omega) \times H^{s}(\Omega)^{d} \times H^{s}(\Omega)$ for a real number $s>\frac{d+1}{2}$. Then, the following error estimate holds between this solution and the solution $\left(\boldsymbol{\omega}_{N}, \boldsymbol{u}_{N}, p_{N}\right)$ of problem (3.10)

$$
\begin{aligned}
\| \boldsymbol{\omega} & -\boldsymbol{\omega}_{N}\left\|_{H(\operatorname{curl}, \Omega)}+\right\| \boldsymbol{u}-\boldsymbol{u}_{N}\left\|_{H(\mathrm{div}, \Omega)}+\right\| p-p_{N} \|_{L^{2}(\Omega)} \\
& \leq c \sum_{k=1}^{K}\left(N^{-s}\left(\|\boldsymbol{\omega}\|_{H^{s}\left(\operatorname{curl}, \Omega_{k}\right)}+\|\boldsymbol{u}\|_{H^{s}\left(\Omega_{k}\right)^{d}}+\|p\|_{H^{s}\left(\Omega_{k}\right)}\right)+N^{-\sigma}\|\boldsymbol{f}\|_{H^{\sigma}\left(\Omega_{k}\right)^{d}}\right) .
\end{aligned}
$$

Estimate (4.19) is fully optimal. Note that this optimality is not obtained for the pressure in most spectral discretizations of the Stokes problem. However, the regularity which is required for this estimate $\left(s>\frac{d+1}{2}\right)$ does not seem reasonable in the general case.

Let $\Pi_{N}^{c}$ denote the orthogonal projection operator from $H_{0}(\mathbf{c u r l}, \Omega)$ onto $\mathbb{C}_{N}$. By combining estimate (4.17) with an interpolation argument, we easily obtain, for all $s \geq 0$,

$$
\left\|\boldsymbol{\omega}-\Pi_{N}^{c} \boldsymbol{\omega}\right\|_{H(\operatorname{curl}, \Omega)} \leq c N^{-s} \sum_{k=1}^{K}\|\boldsymbol{\omega}\|_{H^{s+1}\left(\Omega_{k}\right)^{\frac{d(d-1)}{2}}} .
$$

Note that the replacement of $H^{s}\left(\mathbf{c u r l}, \Omega_{k}\right)$ by $H^{s+1}\left(\Omega_{k}\right)^{\frac{d(d-1)}{2}}$ is due to the fact that no result seems to be known concerning the interpolation of the spaces $H^{s}\left(\mathbf{c u r l}, \Omega_{k}\right)$. A similar projection operator can be used for the approximation of functions in $V$, which leads to the next corollary.

Corollary 4.9. Assume that the data $\boldsymbol{f}$ belong to $H^{\sigma}(\Omega)^{d}$ for a real number $\sigma>\frac{d}{2}$ and also that the solution $(\boldsymbol{\omega}, \boldsymbol{u}, p)$ of problem $(2.9)$ belongs to $H^{s+1}(\Omega)^{\frac{d(d-1)}{2}} \times H^{s}(\Omega)^{d} \times H^{s}(\Omega)$ for a real number $s \geq 0$. Then, the following error estimate holds between this solution and the solution $\left(\boldsymbol{\omega}_{N}, \boldsymbol{u}_{N}, p_{N}\right)$ of problem (3.10)

$$
\begin{aligned}
\| \boldsymbol{\omega} & -\boldsymbol{\omega}_{N}\left\|_{H(\operatorname{curl}, \Omega)}+\right\| \boldsymbol{u}-\boldsymbol{u}_{N}\left\|_{H(\operatorname{div}, \Omega)}+\right\| p-p_{N} \|_{L^{2}(\Omega)} \\
& \leq c \sum_{k=1}^{K}\left(N^{-s}\left(\|\boldsymbol{\omega}\|_{H^{s+1}\left(\Omega_{k}\right) \frac{d(d-1)}{2}}+\|\boldsymbol{u}\|_{H^{s}\left(\Omega_{k}\right)^{d}}+\|p\|_{H^{s}\left(\Omega_{k}\right)}\right)+N^{-\sigma}\|\boldsymbol{f}\|_{H^{\sigma}\left(\Omega_{k}\right)^{d}}\right) .
\end{aligned}
$$

In view of the regularity results stated in Section 2, the assumptions of Corollary 4.9 are now reasonable except in dimension $d=3$ and in the case of a nonconvex polyhedron $\Omega$. However, in this case, it follows from [11] 
and [12] that $\boldsymbol{\omega}$ admits the expansion

$$
\omega=\omega_{r}+\operatorname{grad} S_{\Omega}^{f}
$$

where the regularity of $\boldsymbol{\omega}_{r}$ only depends on that of $\boldsymbol{f}$ while $S_{\Omega}^{\boldsymbol{f}}$ is a linear combination of the singular functions associated with the Laplace operator with Dirichlet boundary conditions in $\Omega$, the coefficients of this combination only depending on $\boldsymbol{f}$. Since grad $S_{\Omega}^{\boldsymbol{f}}$ is curl-free, using a separate approximation of the two terms in this expansion leads to the following estimate which is now valid without assumptions on the regularity of the solution.

Corollary 4.10. Assume that the data $\boldsymbol{f}$ belong to $H^{\sigma}(\Omega)^{d}$ for a real number $\sigma>\frac{d}{2}$. Then, the following error estimate holds between the solution $(\boldsymbol{\omega}, \boldsymbol{u}, p)$ of problem $(2.9)$ and the solution $\left(\boldsymbol{\omega}_{N}, \boldsymbol{u}_{N}, p_{N}\right)$ of problem (3.10)

$$
\begin{aligned}
\left\|\boldsymbol{\omega}-\boldsymbol{\omega}_{N}\right\|_{H(\operatorname{curl}, \Omega)}+\left\|\boldsymbol{u}-\boldsymbol{u}_{N}\right\|_{H(\operatorname{div}, \Omega)}+\| p & -p_{N} \|_{L^{2}(\Omega)} \\
& \leq c N^{-\min \left\{\sigma, \sigma_{\Omega}\right\}}\|\boldsymbol{f}\|_{H^{\sigma}(\Omega)^{d}},
\end{aligned}
$$

where $\sigma_{\Omega}$ is a real number $\geq 1$ only depending on $\Omega$.

\section{SOME NUMERICAL EXPERIMENTS}

Before presenting the numerical experiments, we briefly describe how problem (3.10) is implemented. We only treat the case of dimension $d=2$ for simplicity. Let $\varphi_{j}, 0 \leq j \leq N$, denote the Lagrange polynomials in $\mathbb{P}_{N}(-1,1)$ associated with the nodes $\xi_{j}$. We fix an integer $j^{*}$ between 1 and $N-1$ (usually equal to the integer part of $\frac{N}{2}$ ), define $\mathcal{J}^{*}$ as the set $\{0, \ldots, N\} \backslash\left\{j^{*}\right\}$ and set

$$
\varphi_{j}^{*}(\zeta)=\varphi_{j}(\zeta) \frac{\xi_{j}-\xi_{j^{*}}}{\zeta-\xi_{j^{*}}}, \quad j \in \mathcal{J}^{*}
$$

We now describe the vectors of unknowns. The vector $\Omega^{\diamond}$ of unknowns coresponding to $\boldsymbol{\omega}_{N}$ is made of

- the values of $\boldsymbol{\omega}_{N}$ inside each $\Omega_{k}$, more precisely of $\boldsymbol{\omega}_{N}$ at the nodes $F_{k}\left(\xi_{i}, \xi_{j}\right), 1 \leq i, j \leq N-1$;

- the values of $\boldsymbol{\omega}_{N}$ at the nodes $F_{k}\left( \pm 1, \xi_{j}\right)$ or $F_{k}\left(\xi_{i}, \pm 1\right), 1 \leq i, j \leq N-1$, on each edge of $\Omega_{k}$ which is not contained in $\partial \Omega$ (so that the node is shared by two subdomains);

- and also the values of $\boldsymbol{\omega}_{N}$ at the vertices of the $\Omega_{k}$, one value for each vertex which is not contained in $\partial \Omega$.

Note however that multiplying the vector $\Omega^{\diamond}$ by a matrix $Q_{\boldsymbol{\omega}}$ leads to a vector $\widetilde{\Omega}^{\diamond}=Q_{\boldsymbol{\omega}} \Omega^{\diamond}$ made of $K$ blocks $\Omega_{k}^{\diamond}$ : The coefficients $\boldsymbol{\omega}_{i j}^{k}$ of $\Omega_{k}^{\diamond}$ correspond to the expansion of $\boldsymbol{\omega}_{N}$ on $\Omega_{k}$. If $\zeta_{k}$ and $\eta_{k}$ denote the components of $F_{k}^{-1}$, it reads

$$
\left.\boldsymbol{\omega}_{N}\right|_{\Omega_{k}}(x, y)=\sum_{i=0}^{N} \sum_{j=0}^{N} \omega_{i j}^{k} \varphi_{i} \circ \zeta_{k}(x, y) \varphi_{j} \circ \eta_{k}(x, y)
$$

where all the values of $\boldsymbol{\omega}_{N}$ at the nodes which belong to $\partial \Omega$ are equal to zero.

Similarly, the vector $U$ of unknowns corresponding to $\boldsymbol{u}_{N}$ is made of

- the values of $u_{N x}$ at the nodes $F_{k}\left(\xi_{i}, \xi_{j}\right), 1 \leq i \leq N-1, j \in \mathcal{J}^{*}$, and of $u_{N y}$ at the nodes $F_{k}\left(\xi_{i}, \xi_{j}\right)$, $i \in \mathcal{J}^{*}, 1 \leq j \leq N-1$;

- the values of $u_{N x}$ at the nodes $F_{k}\left(\xi_{i}, \pm 1\right), 1 \leq i \leq N-1$, of edges of $\Omega_{k}$ which are parallel to the $x$-axis and the values of $u_{N y}$ at the nodes $F_{k}\left( \pm 1, \xi_{j}\right), 1 \leq j \leq N-1$, of edges of $\Omega_{k}$ which are parallel to the $y$-axis;

- the values of $u_{N x}$ at the nodes of each edge which are parallel to the $y$-axis and shared by two subdomains and the values of $u_{N y}$ at the nodes of each edge which are parallel to the $x$-axis and shared by two subdomains; 
- and also the appropriate values of $\boldsymbol{u}_{N}$ at the vertices of the $\Omega_{k}$ (the continuity of $\boldsymbol{u}_{N} \cdot \boldsymbol{n}$ implies that the values of $\left.\boldsymbol{u}_{N}\right|_{\Omega_{k}}$ at a vertex $\boldsymbol{a}$ are the same for all $\Omega_{k}$ such that $\boldsymbol{a}$ is a vertex of $\Omega_{k}$ );

- minus $J$ values, one per $\Sigma_{j}$, in order to enforce the conditions (2.3).

There also, multiplying the vector $U$ by a matrix $Q_{\boldsymbol{u}}$ leads to a vector $\widetilde{U}=Q_{\boldsymbol{u}} U$ made of $K$ blocks $U_{k}$ : The coefficients $\boldsymbol{u}_{i j}^{k}$ of $U_{k}$ correspond to the expansion of $\boldsymbol{u}_{N}$ on $\Omega_{k}$, which reads

$$
\begin{aligned}
\left.u_{N x}\right|_{\Omega_{k}}(x, y)=\sum_{i=0}^{N} \sum_{j \in \mathcal{J}^{*}} u_{x, i j}^{k} \varphi_{i} \circ \zeta_{k}(x, y) \varphi_{j}^{*} \circ \eta_{k}(x, y) & \\
\left.u_{N y}\right|_{\Omega_{k}}(x, y) & =\sum_{i \in \mathcal{J}^{*}} \sum_{j=0}^{N} u_{y, i j}^{k} \varphi_{i}^{*} \circ \zeta_{k}(x, y) \varphi_{j} \circ \eta_{k}(x, y),
\end{aligned}
$$

where all the values of $\boldsymbol{u}_{N} \cdot \boldsymbol{n}$ at the nodes which belong to $\partial \Omega$ are equal to zero.

Finally, the vector $P$ of unknowns corresponding to $p_{N}$ is made of $K$ blocks, each of them made of the coefficients of a pseudo-pressure $\tilde{p}_{N}$ at the nodes $F_{k}\left(\xi_{i}, \xi_{j}\right), i \in \mathcal{J}^{*}, j \in \mathcal{J}^{*}$,

$$
\left.\tilde{p}_{N}\right|_{\Omega_{k}}(x, y)=\sum_{i \in \mathcal{J}^{*}} \sum_{j \in \mathcal{J}^{*}} p_{i j} \varphi_{i}^{*} \circ \zeta_{k}(x, y) \varphi_{j}^{*} \circ \eta_{k}(x, y)
$$

where the value of $\tilde{p}_{N}$ at one arbitrary node of one of the $\Omega_{k}$ is taken equal to zero. The function $\tilde{p}_{N}$ vanishes at this node but does no longer belong to $L_{0}^{2}(\Omega)$, however the real pressure $p_{N}$ can easily be recovered in a post-processing step, thanks to the formula

$$
p_{N}(x, y)=\tilde{p}_{N}(x, y)-\frac{1}{\operatorname{meas}(\Omega)}\left(\left(\tilde{p}_{N}, 1\right)\right)_{N}
$$

Problem (3.10) can thus be written equivalently as the square linear system

$$
\left(\begin{array}{ccc}
Q_{\boldsymbol{u}}^{T} A Q_{\boldsymbol{\omega}} & 0 & Q_{\boldsymbol{u}}^{T} B \\
0 & B^{T} Q_{\boldsymbol{u}} & 0 \\
Q_{\boldsymbol{\omega}}^{T} C_{\boldsymbol{\omega}} Q_{\boldsymbol{\omega}} & Q_{\boldsymbol{\omega}}^{T} C_{\boldsymbol{u}} Q_{\boldsymbol{u}} & 0
\end{array}\right)\left(\begin{array}{c}
\Omega^{\diamond} \\
U \\
P
\end{array}\right)=\left(\begin{array}{c}
Q_{\boldsymbol{u}}^{T} F \\
0 \\
0
\end{array}\right)
$$

where, for each matrix $M, M^{T}$ denotes the transposed matrix of $M$. The global matrix is not symmetric, even if the sub-blocks $\left(\begin{array}{cc}0 & Q_{\boldsymbol{u}}^{T} B \\ B^{T} Q_{\boldsymbol{u}} & 0\end{array}\right)$ and $Q_{\boldsymbol{\omega}}^{T} C_{\boldsymbol{\omega}} Q_{\boldsymbol{\omega}}$ are. Note that, up to the multiplicative constant $-\nu^{-1}$, the matrix $C_{\boldsymbol{u}}$ coincides with $A^{T}$.

The choice of system (5.6), which relies on the multiplication by the $Q$-type matrices, yields that now the matrices $A, B, C_{\boldsymbol{\omega}}$ and $C_{\boldsymbol{u}}$ are fully block-diagonal, each block corresponding to one subdomain $\Omega_{k}$.

In what follows, system (5.6) is solved via the GMRES method, so that it has not to be assembled. We also use local preconditioners: Each block which appears in the global matrix in (5.6) is preconditioned by the matrix issued from its incomplete $L U$ factorization. Note finally that, as standard in spectral methods, the tensorization properties of the polynomial spaces yield that each product of one of these blocks, corresponding to the subdomain $\Omega_{k}$, by a vector is realized with $c N^{d+1}$ operations, which highly reduces the cost of the inversion.

We first check the convergence of the discretization. We work with the $L$-shaped domain $\Omega=]-1,1\left[\left[^{2} \backslash\left[0,1\left[{ }^{2}\right.\right.\right.\right.$, divided into three square subdomains

$$
\left.\Omega_{1}=\right]-1,0[\times] 0,1\left[, \quad \Omega_{2}=\right]-1,0\left[^{2}, \quad \Omega_{3}=\right] 0,1[\times]-1,0[,
$$




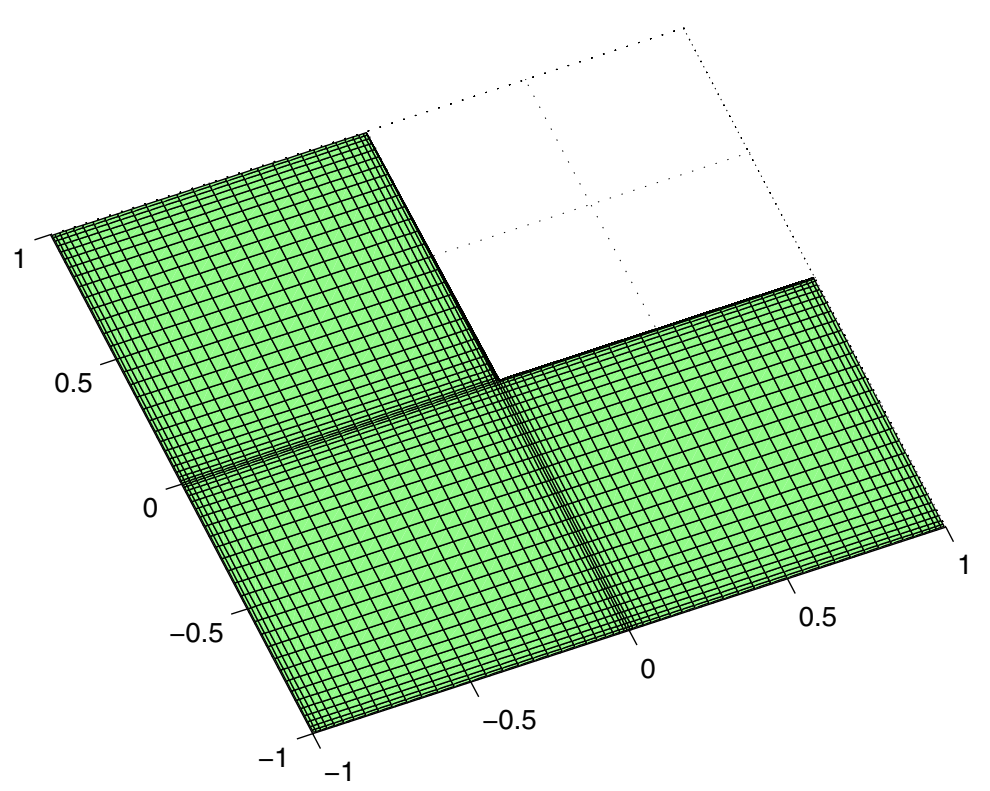

Figure 1. The $L$-shaped domain and its decomposition.

as illustrated in Figure 1. We consider the solution $(\boldsymbol{\omega}, \boldsymbol{u}, p)$ of problem (2.2) given by $\boldsymbol{\omega}=\operatorname{curl} \boldsymbol{u}, \boldsymbol{u}=\mathbf{c u r l} \psi$, with

$$
\psi(x, y)=\sin (\pi x) \sin (\pi y), \quad p(x, y)=x y+\frac{1}{12}
$$

Figure 2 presents the curves of the errors

$$
\left\|\boldsymbol{\omega}-\boldsymbol{\omega}_{N}\right\|_{H(\operatorname{curl}, \Omega)}, \quad\left\|\boldsymbol{u}-\boldsymbol{u}_{N}\right\|_{H(\operatorname{div}, \Omega)}, \quad\left\|p-p_{N}\right\|_{L^{2}(\Omega)},
$$

in logarithmic scale, as a function of $N$, for $N$ varying from 5 to 30 . As can be expected from Theorem 4.8 , the convergence is exponential for this solution, and the slope for the error on the pressure is exactly the same as for the two other unknowns.

We now present two numerical experiments, again in dimension $d=2$ and in the case where the data $\boldsymbol{f}=\left(f_{x}, f_{y}\right)$ are given by $f_{x}=y, f_{y}=0$, but the homogeneous boundary condition in the fourth line of (2.2) is replaced by

$$
\boldsymbol{u} \cdot \boldsymbol{n}=g \text { on } \partial \Omega,
$$

where $g$ belongs to $L^{2}(\partial \Omega)$ and has a null integral on $\partial \Omega$. Indeed, this situation is slightly more realistic in applications: For instance, we consider below the case of a Poiseuille flow where no vorticity is induced at the walls due to the geometry of the domain. We refer to [4], Section 5, for the rather simple extension of the previous analysis to the new boundary condition (5.9), that we do not give here for brevity. For both experiments, we work with a Poiseuille type flow and in the case where $g$ is equal to zero when $\boldsymbol{n}=(0, \pm 1)$ is parallel to the $y$-axis.

The first numerical experiment still deals with the $L$-shaped domain $\Omega=]-1,1\left[^{2} \backslash\left[0,1\left[^{2}\right.\right.\right.$ and its decomposition (5.7), as drawn in Figure 1. The datum $g$ is given by

$$
\begin{aligned}
& g(-1, y)=y^{2}-1,-1 \leq y \leq 1, \quad g(0, y)=0,0 \leq y \leq 1, \\
& \text { and } \quad g(1, y)=-8 y(1+y),-1 \leq y \leq 0
\end{aligned}
$$




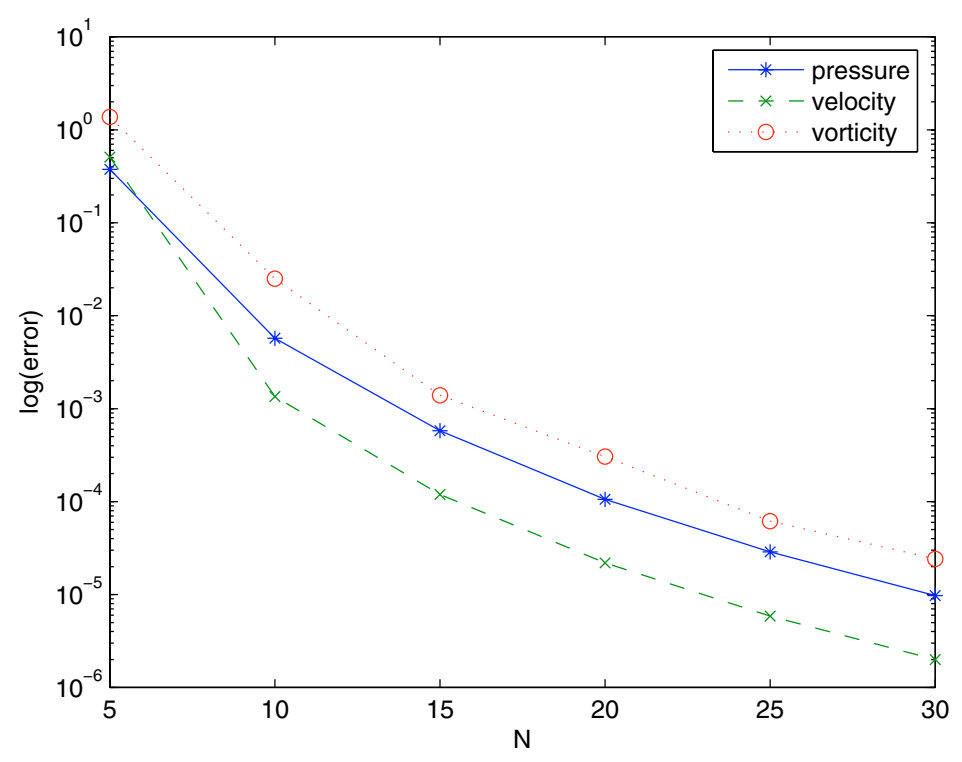

Figure 2. The error curves for the solution obtained from (5.8).

Note that, even if the function $g$ is very smooth, the corresponding solution is not since the domain $\Omega$ is not convex. Figure 3 presents, from left to right and from top to bottom, the values of the vorticity, the two components of the velocity and the pressure for the discrete solution obtained with $N=35$.

The second numerical experiment deals with the multiply-connected domain

$$
\Omega=]-2,2\left[\left[^{2} \backslash[-1,1]^{2}\right.\right.
$$

divided in an obvious way into four equal squares and four equal rectangles, as illustrated in Figure 4, and with the cut $\Sigma_{1}$ equal to $] 1,2[\times\{1\}$.

The datum $g$ is now given by

$$
\begin{gathered}
g(-2, y)=\left\{\begin{array}{ll}
y^{2}-1, & -1 \leq y \leq 1, \\
0, & 1 \leq|y| \leq 2,
\end{array}, \quad g( \pm 1, y)=0,-1 \leq y \leq 1,\right. \\
\text { and } g(2, y)= \begin{cases}-8(1+y)(2+y), & -2 \leq y \leq-1, \\
0, & -1 \leq y \leq 2 .\end{cases}
\end{gathered}
$$

Figure 5 presents, from left to right and from top to bottom, the values of the vorticity, the two components of the velocity and the pressure for the discrete solution obtained with $N=35$.

The formulation that we have used leads to very efficient simulations of viscous flows, when both spectral or spectral element discretizations are used. The extension of this work to the full Navier-Stokes equations has begun. The extension to mixed boundary conditions is also under consideration but seems less natural. 

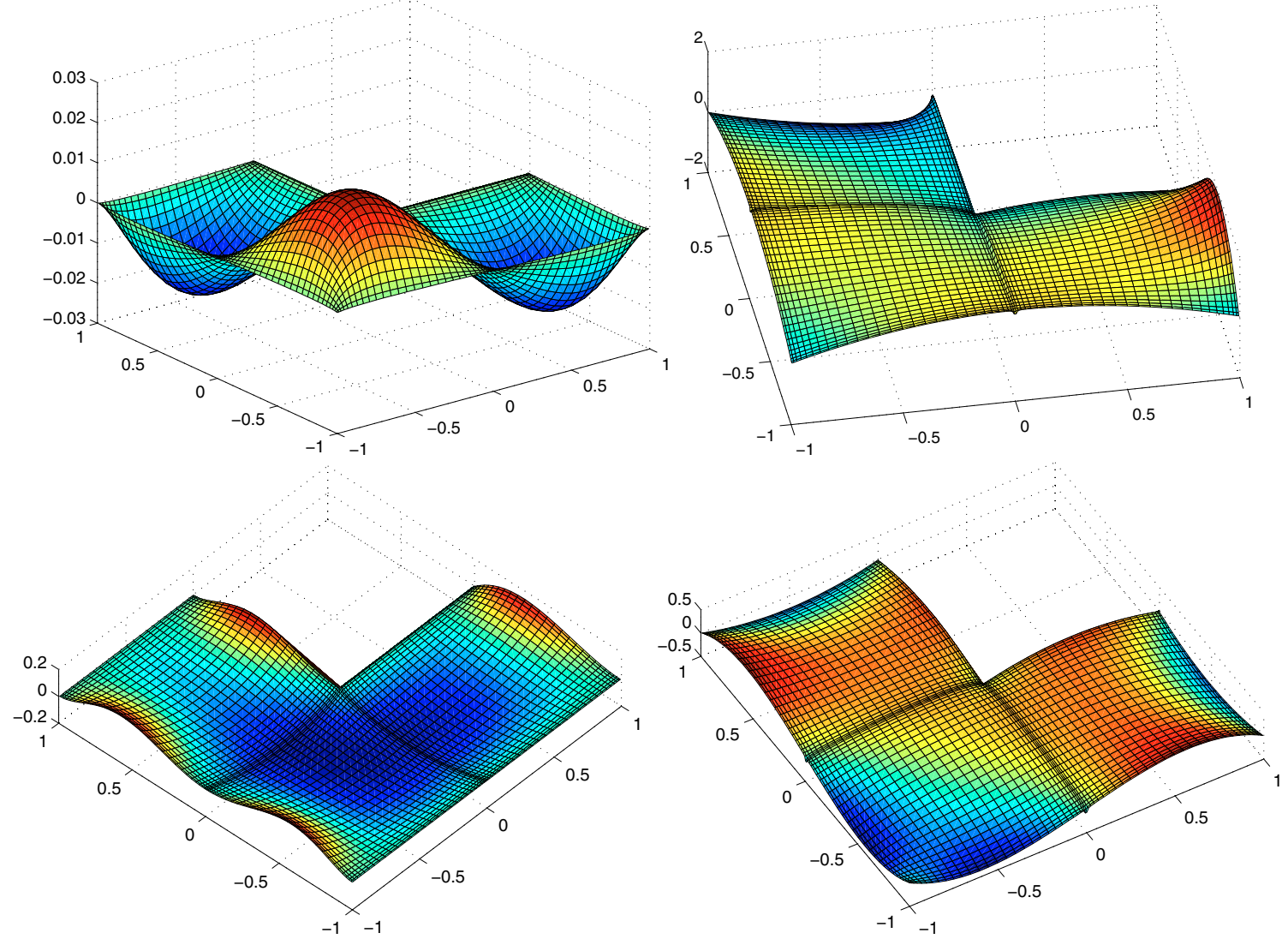

FigURE 3. The values of the discrete solution issued from (5.10).

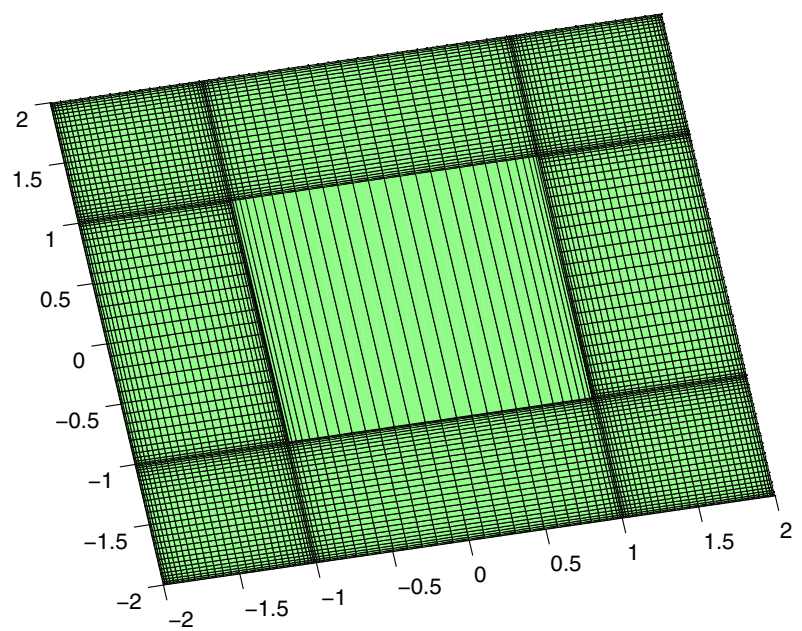

FIGURE 4 . The square ring domain and its decomposition. 

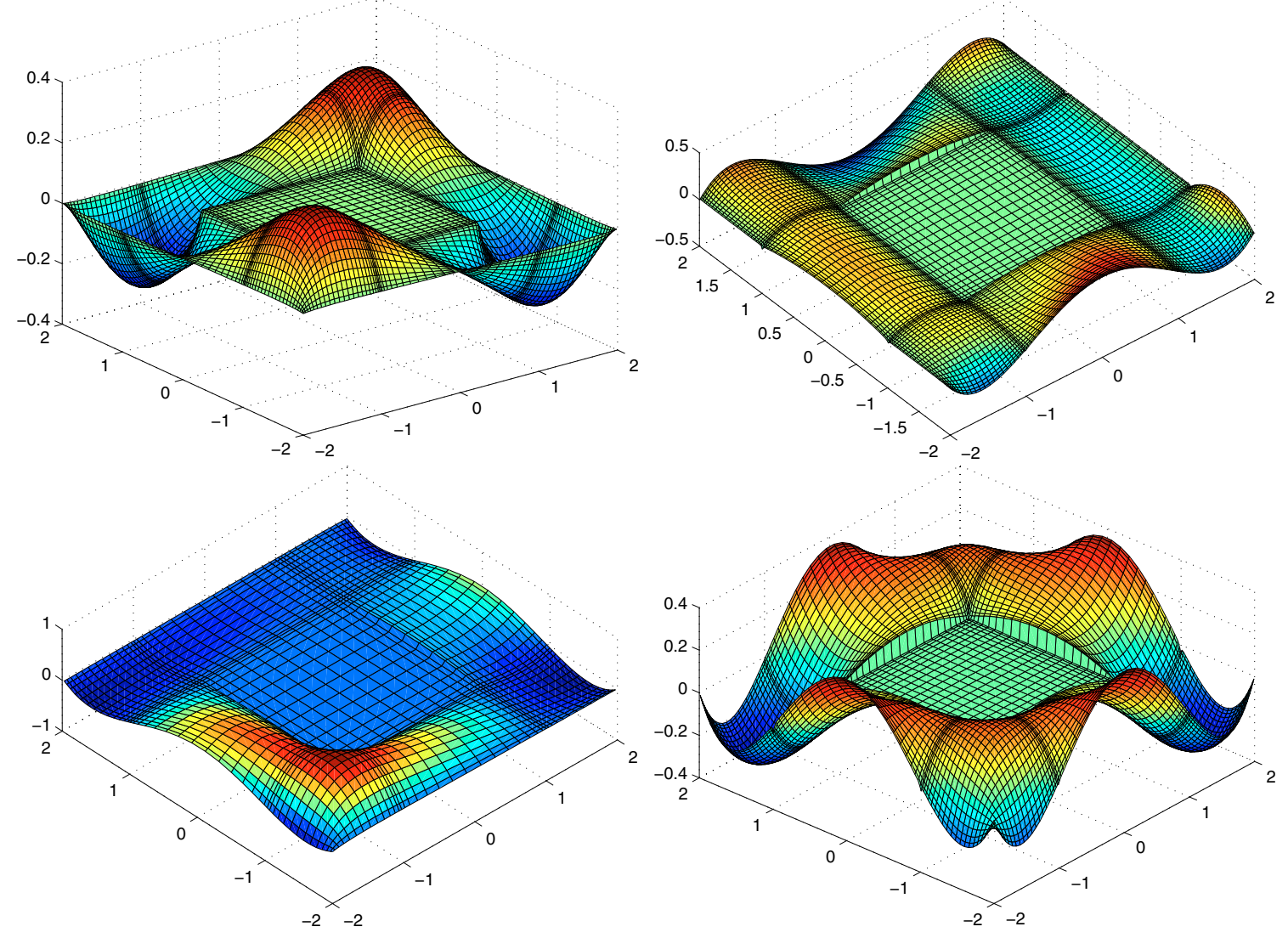

FIGURE 5. The values of the discrete solution issued from (5.12).

Acknowledgements. The first author is very grateful toward Professor Fatma Zohra Nouri for her help concerning this work. The second and third authors wish to thank her for her invitation to the University Badji-Mokhtar at Annaba. They are also deeply grateful toward Pascal Joly for his help concerning the numerical experiments.

\section{REFERENCES}

[1] M. Amara, D. Capatina-Papaghiuc, E. Chacón-Vera and D. Trujillo, Vorticity-velocity-pressure formulation for Navier-Stokes equations. Comput. Vis. Sci. 6 (2004) 47-52.

[2] C. Amrouche, C. Bernardi, M. Dauge and V. Girault, Vector potentials in three-dimensional nonsmooth domains. Math. Method. Appl. Sci. 21 (1998) 823-864.

[3] F. Ben Belgacem and C. Bernardi, Spectral element discretization of the Maxwell equations. Math. Comput. 68 (1999) 14971520 .

[4] C. Bernardi and N. Chorfi, Spectral discretization of the vorticity, velocity and pressure formulation of the Stokes problem. SIAM J. Numer. Anal. 44 (2006) 826-850.

[5] C. Bernardi and Y. Maday, Spectral Methods, in the Handbook of Numerical Analysis V, P.G. Ciarlet and J.-L. Lions Eds., North-Holland (1997) 209-485.

[6] C. Bernardi, M. Dauge and Y. Maday, Polynomials in the Sobolev world. Internal Report, Laboratoire Jacques-Louis Lions, Université Pierre et Marie Curie (2003).

[7] C. Bernardi, V. Girault and P.-A. Raviart, Incompressible Viscous Fluids and their Finite Element Discretizations, in preparation.

[8] J. Boland and R. Nicolaides, Stability of finite elements under divergence constraints. SIAM J. Numer. Anal. 20 (1983) 722-731. 
[9] A. Buffa and P. Ciarlet, Jr., On traces for functional spaces related to Maxwell's equations. Part II: Hodge decompositions on the boundary of Lipschitz polyhedra and applications. Math. Method. Appl. Sci. 24 (2001) 31-48.

[10] A. Buffa, M. Costabel and M. Dauge, Algebraic convergence for anisotropic edge elements in polyhedral domains. Numer. Math. 101 (2005) 29-65.

[11] M. Costabel and M. Dauge, Espaces fonctionnels Maxwell: Les gentils, les méchants et les singularités, Web publication (1998) http://perso.univ-rennes1.fr/monique.dauge.

[12] M. Costabel and M. Dauge, Computation of resonance frequencies for Maxwell equations in non smooth domains, in Topics in Computational Wave Propagation, M. Ainsworth, P. Davies, D. Duncan, P. Martin and B. Rynne Eds., Springer (2004) $125-161$.

[13] F. Dubois, Vorticity-velocity-pressure formulation for the Stokes problem. Math. Meth. Appl. Sci. 25 (2002) $1091-1119$.

[14] F. Dubois, M. Salaün and S. Salmon, Vorticity-velocity-pressure and stream function-vorticity formulations for the Stokes problem. J. Math. Pure. Appl. 82 (2003) 1395-1451.

[15] V. Girault and P.-A. Raviart, Finite Element Methods for Navier-Stokes Equations, Theory and Algorithms. Springer-Verlag (1986).

[16] J.-C. Nédélec, Mixed finite elements in $\mathbb{R}^{3}$. Numer. Math. 35 (1980) 315-341.

[17] P.-A. Raviart and J.-M. Thomas, A mixed finite element method for second order elliptic problems, in Mathematical Aspects of Finite Element Methods, I. Galligani and E. Magenes Eds., Lect. Notes Math. 606, Springer-Verlag (1977) 292-315.

[18] S. Salmon, Développement numérique de la formulation tourbillon-vitesse-pression pour le problème de Stokes. Ph.D. thesis, Université Pierre et Marie Curie, Paris (1999).

To access this journal online:

www.edpsciences.org 